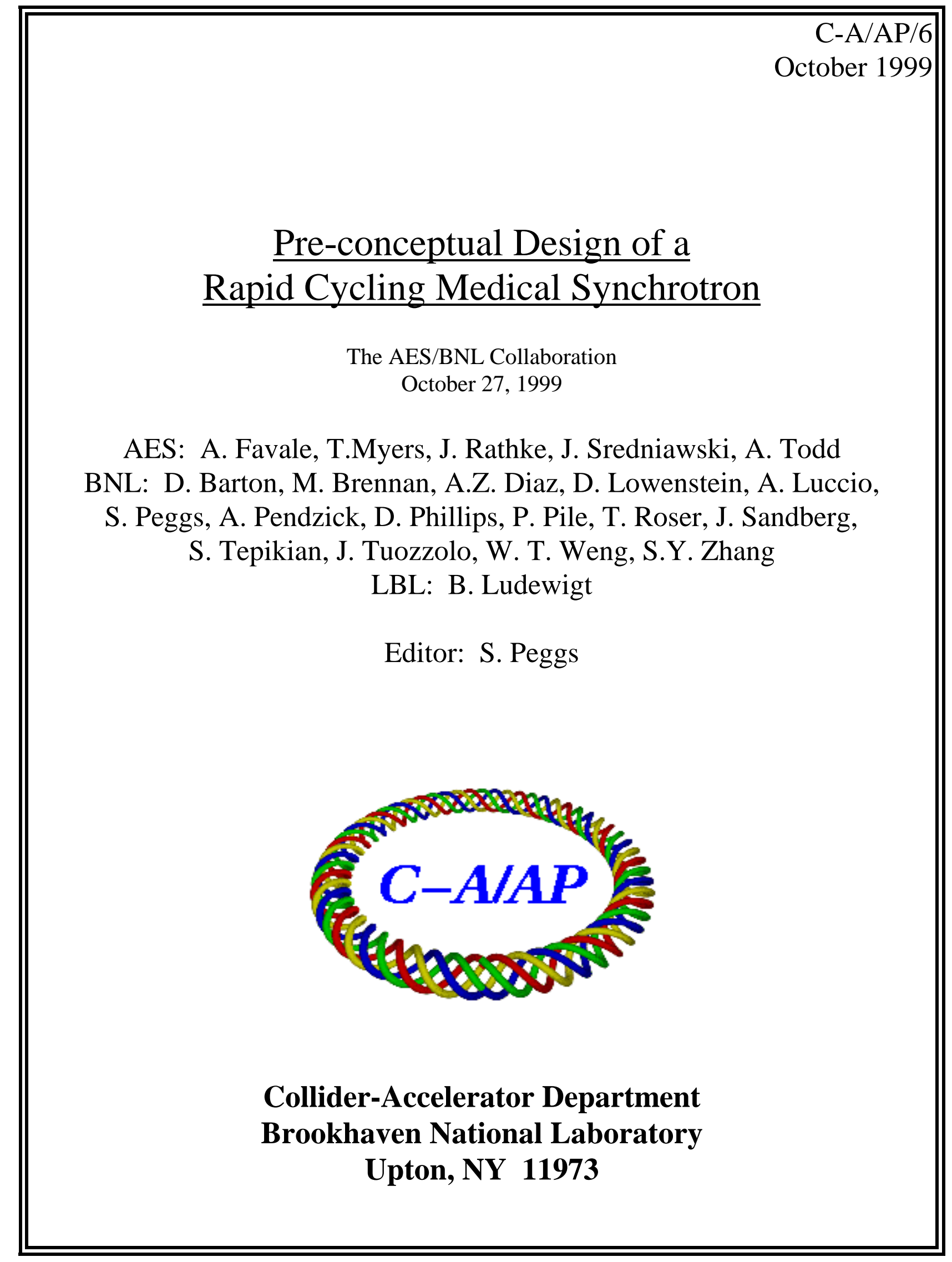




\title{
Pre-conceptual Design of a Rapid Cycling Medical Synchrotron
}

\author{
The AES/BNL collaboration \\ October 27, 1999
}

AES: A. Favale, T. Myers, J. Rathke, J. Sredniawski, A. Todd

BNL: D. Barton, M. Brennan, A.Z. Diaz, D. Lowenstein, A. Luccio, S. Peggs,

A. Pendzick, D. Phillips, P. Pile, T. Roser, J. Sandberg, S. Tepikian,

J. Tuozzolo, B. Weng, S.Y. Zhang

LBL: B. Ludewigt

Editor: S. Peggs

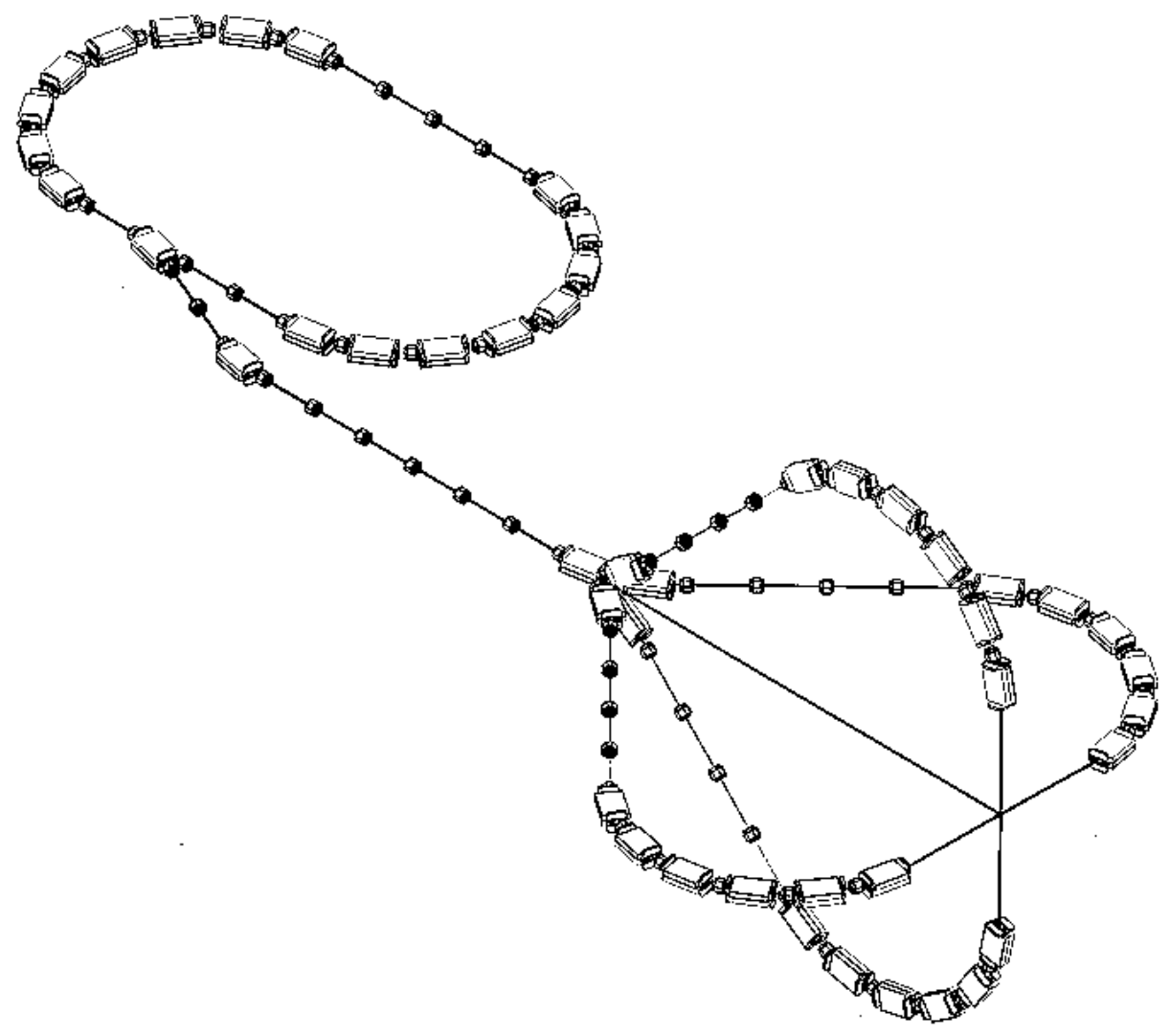




\section{Contents}

1 Introduction and Overview 5

1.1 Key features . . . . . . . . . . . . . . . 6

1.2 Performance upgrades . . . . . . . . . . . . . 7

2 Injector 9

2.1 Proton Source \& Low Energy Beam Transport (LEBT) . . . . 10

2.2 RF Accelerator . . . . . . . . . . . . . . . . . . . 11

2.3 High Energy Beam Transport (HEBT) . . . . . . . . . . . . 14

2.4 Control System . . . . . . . . . . . . . . . . . . . 14

2.5 Support Subsystems . . . . . . . . . . . . . . . . 15

$\begin{array}{lll}3 & \text { Synchrotron } & 17\end{array}$

3.1 Optics . . . . . . . . . . . . . . . . . 18

3.2 Magnets . . . . . . . . . . . . . . . . . . . . 22

3.3 Main power supplies and ramping . . . . . . . . . . . 23

3.4 Radio Frequency . . . . . . . . . . . . . . . . . 28

3.5 Longitudinal dynamics . . . . . . . . . . . . . . . . . . 30

3.6 Accelerator controls . . . . . . . . . . . . . . 36

4 Beam delivery $\quad 37$

4.1 Switch yard and transport line . . . . . . . . . . 37

4.2 Planar gantry . . . . . . . . . . . . . . . . 40

4.3 Variable focusing nozzle . . . . . . . . . . . . . . 41

4.4 Treatment planning and patient safety . . . . . . . . . 43 


\begin{abstract}
The Rapid Cycling Medical Synchrotron (RCMS) delivers more than $3 \times 10^{12}$ protons per minute at a maximum extraction energy of $270 \mathrm{MeV}$, with single turn "fast extraction" at a repetition rate of $15 \mathrm{~Hz}$. Upgrade paths are available to deliver substantially more protons per minute, to increase the extraction energy to $320 \mathrm{MeV}$, and to enable "slow extraction".
\end{abstract}

The main advantages for proton therapy are:

- easy pulse-by-pulse adjustment of energy and intensity

- less beam on more frequent cycles in digital dose delivery

- upgradability: wobbling, scanning, proton radiography

- identical simple light magnets in both synchrotron and gantry

- easy installation and maintenance with small modular components

Three key design choices account for these advantages - fast extraction, rapid cycling, and strong focussing. At all levels the underlying design goals are:

- reliability, simplicity, and modularity

- early and economic patient treatment

In its initial configuration the RCMS delivers fast extracted beam into a double scattering system at the end of a fixed beamline, or at the end of a gantry. The energy of the extracted beam is easily adjusted to any desired value between 70 $\mathrm{MeV}$ and $270 \mathrm{MeV}$.

Three dimensional voxel scanning is also best performed using fast extraction. Nonetheless, the RCMS can easily be upgraded to include slow extraction capabilities, if this is deemed necessary for scanning or for other purposes.

Less beam is accelerated per cycle in a rapid cycling sycnchrotron, making the accelerator performance more reliable, the design simpler, and the commissioning more rapid. Fast extraction and rapid cycling together make possible dose delivery with digital accuracy.

Strong focusing is achieved by building the synchrotron from "FODO cell" modules containing dipole (bending) magnets, and quadrupole (focusing) magnets. 
Each dipole is short, permitting a rectangular design with a small cross section that is easy to construct. The dipole is light in weight, and economical in operation.

The same dipole and quadrupole magnets are used in the fixed beamlines and gantries. The planar gantry design is simple and light, with flexible FODO cell matched achromatic optics.

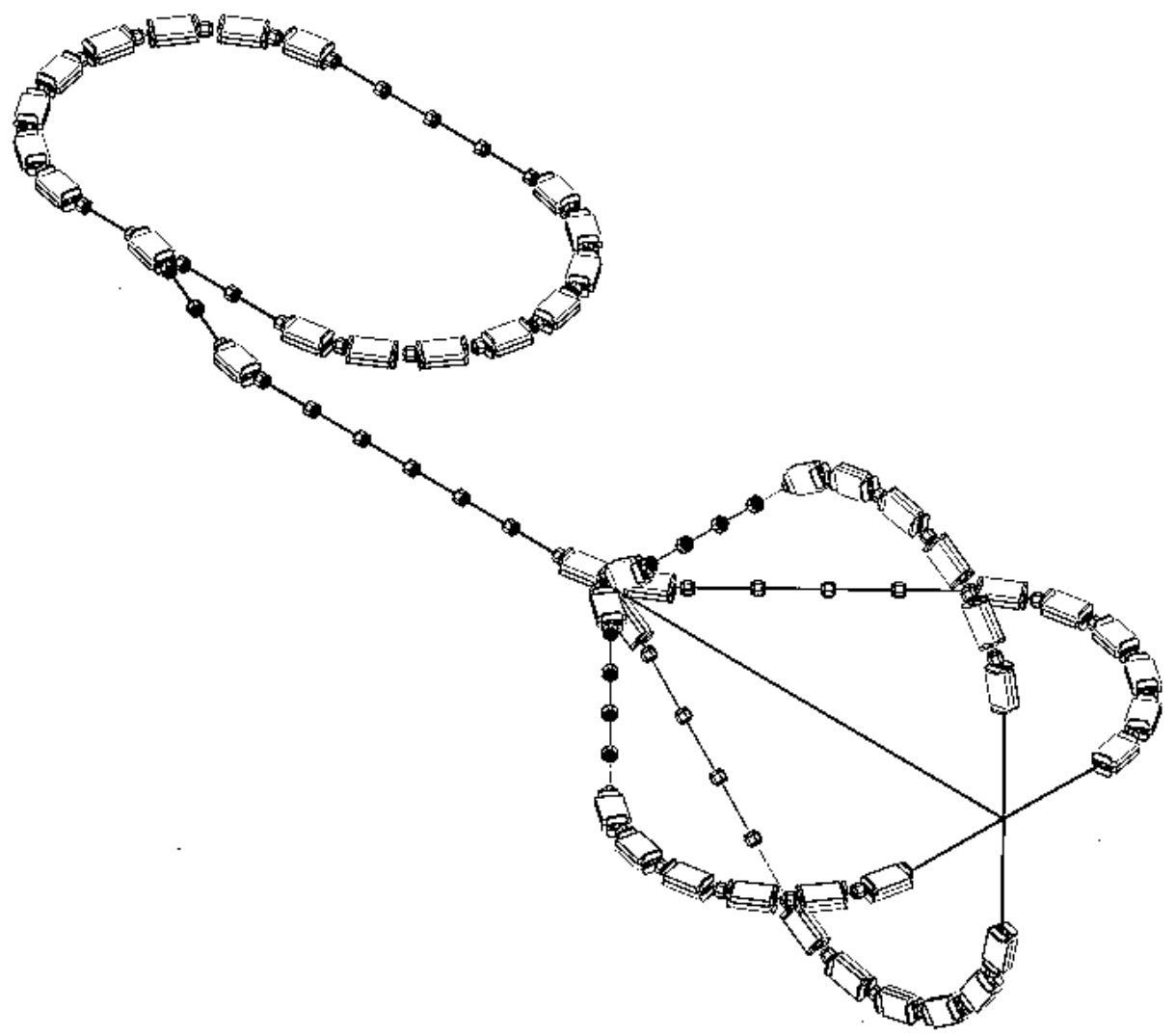

Figure 1: The racetrack footprint of the RCMS, with two straight sections for injection, extraction, and RF systems, and two 180 degree arcs. The geometry of the planar gantry is also shown, at four rotational angles. 


\section{Introduction and Overview}

A synchrotron is the machine of choice for proton therapy. The most significant advantage over a linac or a cyclotron is that an appropriately designed synchrotron can easily produce a beam whose output energy is continuously variable within wide limits, a capability necessary for contemporary beam delivery modalities. Linacs and cyclotrons can produce more intense beams, but a well-designed synchrotron - especially a rapid cycling synchrotron - can easily provide enough beam intensity for a therapy facility.

The performance parameters of the RCMS design, displayed in Table 1 and elsewhere below, are realistic. They do not approach the performance limits that have been achieved in other accelerators. The RCMS is also conservative in its adoption of technologies that are well-established by successful use elsewhere. The design emphasizes simplicity and modularity, for example by minimizing the number of different component types, so that many parts are interchangeable.

\begin{tabular}{|lc|}
\hline Property [units] & Value \\
\hline & \\
\hline Injection kinetic energy [MeV] & 7 \\
Maximum extraction kinetic energy [MeV] & 270 \\
Repetition rate, $f_{\text {rep }}[\mathrm{Hz}]$ & 15 \\
Protons per bunch, $N$ & $3.3 \times 10^{9}$ \\
Accelerated flux, $R$ [protons/min] & $3.0 \times 10^{12}$ \\
Circumference, $C[\mathrm{~m}]$ & 28.6 \\
\hline
\end{tabular}

Table 1: Primary parameters of the Rapid Cycling Medical Synchrotron.

The space requirements for the racetrack synchrotron shown in Figure 1 are modest - the circumference is only 28.6 meters. It is likely that the beam transport system will occupy much more floor space, as at other facilities. In principle the circumference could be reduced by a few percent, despite the increased installation and maintenance difficulties which would ensue. The cost saving achieved by reducing the circumference would be small or negligible, since the total length of all the dipole magnets - the most costly magnets - is unaltered.

In contrast, a careful design of the beam delivery system, including the gantries, can result in large cost savings. The fixed beam lines and the gantries of the RCMS, described below, are simple, cheap, and elegant. Their optics are 
matched and achromatic, using the same small and light magnets that are used in the synchrotron. Unlike the synchrotron, there is a considerable trade-off between cost and space in the design of the gantry. A "corkscrew" gantry saves building space at the expense of needing almost twice as many dipoles as the "planar" gantry described below. It is assumed in what follows that, while space constraints are important, they are not of the same paramount (and sometimes competing) importance as reliability, simplicity, and modularity.

\section{$1.1 \quad$ Key features}

Three features set the RCMS apart in its ability to achieve its design goals: fast extraction, rapid cycling, and strong focusing.

\section{Fast extraction and rapid cycling - a digital approach to dose delivery.}

In nominal operation, the rapid cycling RCMS uses single turn fast extraction to deliver beam into a double scattering system. (Nonetheless, an available upgrade path allows beam to be extracted from the RCMS in a resonant "slow extraction" mode, on a time scale of order one second.)

Fast extraction and rapid cycling are also optimal for scanning large tumor volumes by delivering one beam bunch after another to neighboring voxels. This beam delivery method is a very promising digital approach to realizing the full potential of proton therapy. Fast extraction is simple, and has the great advantage that a single shot of beam can be fast extracted at arbitrary energy simply by changing the firing time of the extraction kicker.

Unlike slow spill, fast extraction imposes no special requirements on the good-field aperture and on field stability at extraction time. Fast extraction is very insensitive to noise and fluctuations. Painting the beam over a tumor volume during slow spill requires a very uniform spill and/or a feed-back system; it is hard in practice to avoid slow spill fluctuations of the order of ten per cent or more.

A slow cycle also demands that a lot of protons occupy the accelerator simultaneously, in order to achieve acceptably short treatment times. Slow extraction therefore carries the threat of suddenly dumping a lot of beam into the patient. Rapid cycling allows the average rate requirements to be met with modest intensities per cycle, also eliminating intensity-dependent problems such as coherent instabilities and space-charge effects.

The high level of repeatability that comes with rapid cycling provides a natural way to ensure delivery of the intended number of protons on each cycle. 
A rare abnormal cycle with a beam intensity that falls outside tight intensity specifications is easily expelled from the synchrotron - and not delivered to the patient.

\section{Strong focusing.}

The magnet layout includes regularly spaced quadrupoles that alternately focus and defocus the beam. This results in "strong focusing" in both transverse planes. The space between quadrupoles is either magnetically empty, or contains a single bending dipole. Straight and bending "half-cells" are assembled to make the racetrack layout shown in Figure 1.

The horizontal and vertical beam sizes are naturally small, allowing the use of small aperture magnets that are small, light, and cheap. Only one kind of dipole magnet and one kind of quadrupole magnet are necessary for the synchrotron, and for the beam transport and gantry systems.

\subsection{Performance upgrades}

A maximum kinetic energy of $270 \mathrm{MeV}$ is sufficient for proton therapy because it allows the beam to penetrate most of the way through most human bodies in any direction perpendicular to the long axis. However, the RCMS design includes provision for a higher kinetic energy of $320 \mathrm{MeV}$ to permit proton computed tomography, which requires penetration all the way through the body. Proton $\mathrm{CT}$ is like X-ray CT except that the images are based not on X-ray attenuation measurements but on measurements of proton energy loss or residual range for multiple trajectories through the body. The $19 \%$ change in kinetic energy increases the range by $31 \%$ while raising the maximum beam momentum by $10 \%$ and the size of the synchrotron by only about $6 \%$; the cost impact is modest.

Proton CT can produce medical images of quality comparable to conventional X-ray CT machines while delivering significantly lower doses. The ability to acquire high-quality images quickly using the same beam and with the patient positioned in the same way as for treatment could be a significant addition to the capability of the proton therapy facility. Such images could be very useful for verifying correct patient positioning; the ability to monitor changes that may occur during the course of treatment could also prove valuable. The proton CT capabilities provided in an RCMS upgrade represent a potentially interesting research opportunity.

The RCMS achieves an extracted beam intensity of more than $3 \times 10^{12}$ protons per minute, sufficient to supply the needs of a large therapy facility. Although this is small compared to the intensity achieved by high-energy physics 
synchrotrons like the BNL AGS, it is not trivial to achieve in a slow cycling lowenergy medical synchrotron with a low injection energy.

Higher intensities may prove valuable for shortening treatment times and/or for supplying beam to additional treatment rooms in the future. The high injection energy of $7 \mathrm{MeV}$ and the rapid cycling rate of $15 \mathrm{~Hz}$ ensure that the RCMS will not constrain either the size of a tumor that can be treated, or the scope of the facility. 


\section{Injector}

The first beam line component of the RCMS system is the injector subsystem (hereafter called the "injector"). This subsystem serves as the proton source for injection into the synchrotron subsystem. The baseline configuration selected for this report is a linear accelerator (linac) comprised of an multi-cusp plasma type proton source, and a series of RF powered accelerating cavities that serve to group the protons into bunches and accelerate them to a high enough energy for injection into the synchrotron. In addition to these linac components, the injector subsystem contains major support subsystems that provide vacuum, cooling, operational control, and structural support and alignment of the linac components.

\begin{tabular}{|lc|}
\hline Property [units] & Value \\
\hline & \\
\hline Injector Subsystem final energy [MeV] & 7.0 \\
Injector Subsystem Delivered Current [mA] & 2.1 \\
Pulse Rate [Hz] & 15 \\
Pulse Length [nsec] & 250 \\
Energy Spread [keV] & \pm 32 \\
Transverse emittance $[\mu \mathrm{m}]$ & 0.3 \\
\hline
\end{tabular}

Table 2: Top level requirements of the injector system.

Initial system optimization studies of the RCMS system indicate that the top-level operational requirements of the injector system are those listed in Table 2. A comparison of these requirements with established operational linac capabilities worldwide indicates that the RCMS injection subsystem can be easily designed from existing operational systems which supply similar, or more complex, beams. Further, the RCMS injector subsystem should be able to achieve high availability based on the excellent operational experience with existing linacs in this power class.

The selected final energy of $7 \mathrm{MeV}$ offers several benefits over lower injection energies. The protons are more dynamically rigid, ameliorating the disruptive effects of the space charge forces. In conjunction the high injection energy dictates a smaller frequency swing of the RF subsystem in the synchrotron, simplifying the design of the ring magnets and minimizing the overall cost of the entire synchrotron system. 


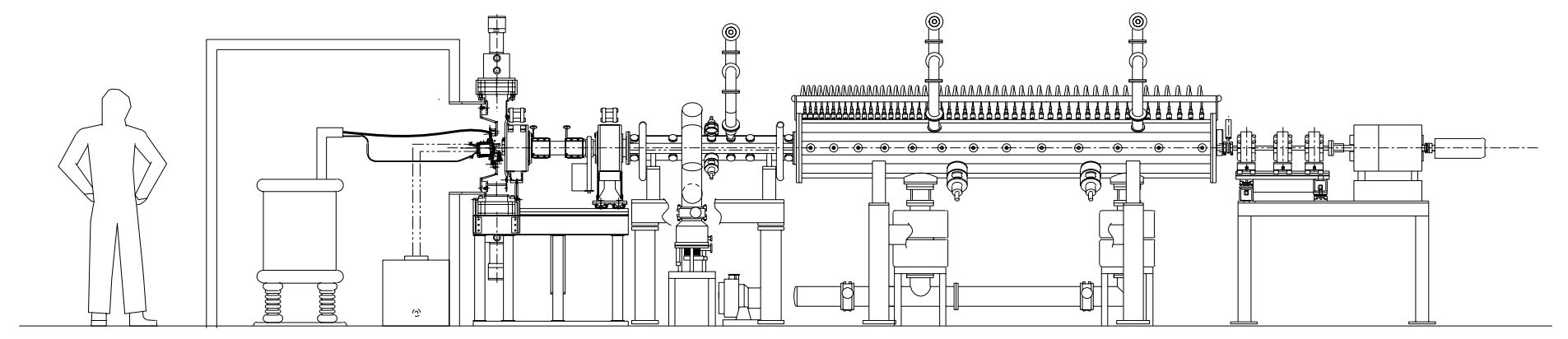

Figure 2: Baseline injector subsystem side elevation sketch identifying major beam line components.

The injector subsystem configuration selected to provide the required parameters is depicted in Figure 2. It is comprised of a RF driven multi-cusp plasma source and low energy beam transport section, a radio-frequency quadrupole (RFQ), a single tank Alvarez type drift tube linac (DTL), an output transport section, a three magnet transport section, and a bending magnet. Final insertion into the ring will be accomplished by an injection kicker subsystem of the ring subsystem that will effectively select 250 nano-second sections from the longer linac pulses. The un-used portion of the linac beam will be dumped in the kicker system.

\subsection{Proton Source \& Low Energy Beam Transport (LEBT)}

The baseline proton source is a multi-cusp magnetically confined configuration employing a hydrogen plasma. The hydrogen plasma provides the ions that are extracted from the plasma chamber by a set of electrodes at an energy of 30 $\mathrm{keV}$. The low energy proton beam emerging from the ion source is divergent in nature. It is then magnetically focussed by a pair of solenoid electromagnets for acceptance into the next section, the RFQ.

The plasma of the ion source requires a source of energy to break down the hydrogen gas into positive and negative ions. This is provided by a low frequency $(2 \mathrm{MHz}) \mathrm{RF}$ source rated at $40 \mathrm{~kW}$ pulsed duty. In addition, there are power supplies required to provide the accelerating voltage across the electrodes, as well as a source of hydrogen gas for the plasma, and associated controls. The major properties of the ion source and LEBT are listed in Table 3. 


\begin{tabular}{|lc|}
\hline Property [units] & Value \\
\hline & \\
Injector Subsystem Delivered Current [mA] & 2.1 \\
Pulse Rate [Hz] & 15 \\
Pulse Length $[\mu \mathrm{s}]$ & 5 \\
Transverse emittance [ $\mu \mathrm{m}]$ & $<0.1$ \\
Overall Length Power Supplies, Source and LEBT [m] & 3.22 \\
\hline
\end{tabular}

Table 3: Major properties of the ion source and Low Energy Beam Transport.

\subsection{RF Accelerator}

The RF accelerator consists of two accelerating structures: an RFQ and a DTL. Also included as part of the RF accelerator WBS is the RF power to drive these structures.

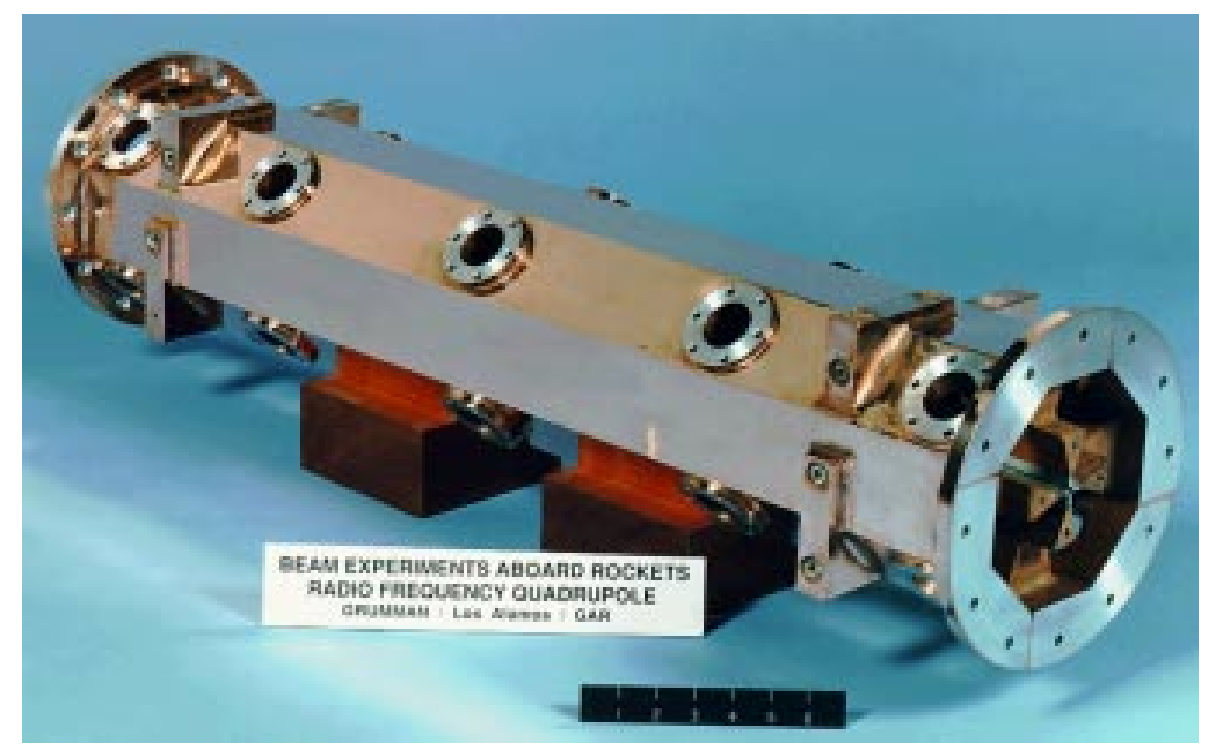

Figure 3: Radio Frequency Quadrupole developed for the Beam Experiment Aboard Rocket. 
The first structure, the RFQ, is envisioned as a standard single physical segment vane-type configuration. The RFQ will accelerate the ion source output beam of $30 \mathrm{keV}$ to a final energy of $1.66 \mathrm{MeV}$. The protons are initially bunched in the front section of the RFQ, and thereafter accelerated up to final energy.

High availability is expected from this device based on an extensive operational database of very similar RFQs. One such system, shown in Figure 3, has been operated for many years in the AES laboratory and has proven to be extremely reliable. It requires approximately $180 \mathrm{~kW}$ of pulsed RF power at $425 \mathrm{MHz}$. The major properties of the RCMS RFQ are listed in Table 4.

\begin{tabular}{|lc|}
\hline Property [units] & Value \\
\hline & \\
RFQ Input Energy [keV] & 30 \\
Nominal Input Current [mA] & $0-10$ \\
Transmission & $90 \%$ \\
Accelerating Frequency [Hz] & 425 \\
Kilpatrick Factor & 1.664 \\
Output Energy [MeV] & 1.66 \\
RF Power Required (Beam \& Cu) [kW] & 154 \\
Overall Length [cm] & 127.0 \\
& \\
\hline
\end{tabular}

Table 4: Major properties of the Radio Frequency Quadrupole (RFQ).

The next accelerating element is a single tank classic Alvarez type DTL. Figure 4 depicts a view inside a small DTL tank. The DTL will accelerate the beam from the RFQ output energy of $1.66 \mathrm{MeV}$ to the final injector subsystem energy of $7.0 \mathrm{MeV}$. The DTL will require $51 \beta \lambda$ accelerating cells employing a FODO focusing lattice. Individual drift tube magnets will be two centimeter long permanent magnets with a strength of $215 \mathrm{~T} / \mathrm{m}$. The DTL tank will be a ramped gradient design (from 1.8 to $3.0 \mathrm{MV} / \mathrm{m}$ ) with a total length of 3.160 meters, requiring $450 \mathrm{~kW}$ of RF power. The major properties of the DTL are listed in Table 5.

Existing DTLs, like the one depicted in Fig. 4, provide emittances far lower than the RCMS requirements. The DTL in Fig. 4 routinely produced an output transverse rms emittance of $0.1 \mu \mathrm{m}$ for a $20 \mathrm{~mA}$ beam (at $1.76 \mathrm{MeV}$ ). This is significantly better than what is required for the RCMS. At the lower current for RCMS, the emittance will be even better. 


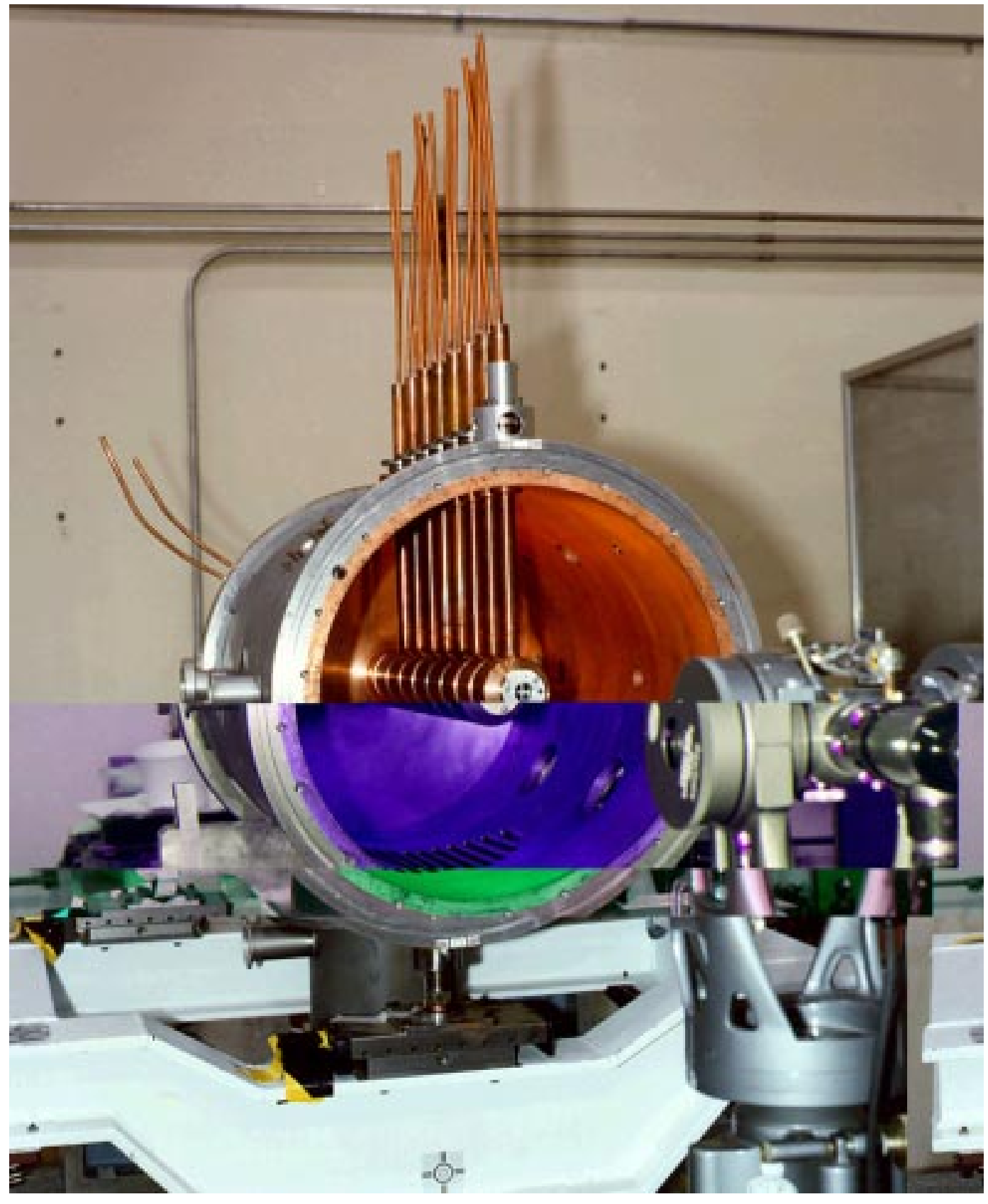

Figure 4: Internal DTL Details (425 MHz). 


\begin{tabular}{|lc|}
\hline Property [units] & Value \\
\hline & \\
\hline DTL Input Energy [MeV] & 1.66 \\
Nominal Input Current [mA] & 2.2 \\
Transmission & $99.9 \%$ \\
Accelerating Frequency [MHz] & 425 \\
Focusing Lattice & $\beta \lambda-$ FODO \\
Magnet Strength [T/m] & 215 \\
Magnet Length [m] & .02 \\
No. of Drift Tubes & 50 \\
Output Energy [MeV] & 7.02 \\
Transverse emittance (rms) [ $\mu \mathrm{m}]$ & 0.3 \\
RF Power Required (Beam \& Cu) $[\mathrm{kW}]$ & 450 \\
Overall Length [m] & 3.16 \\
& \\
\hline
\end{tabular}

Table 5: Major properties of the Drift Tube Linac (DTL).

\subsection{High Energy Beam Transport (HEBT)}

The HEBT subsystem includes the beam transport tube with beam focusing quadrupole magnets, a bending magnet, and a beam dump. The quadrupoles maintain the beam size and shape during transport, while the bending magnet serves to direct the proton beam into the synchrotron injection septum. The final angle of the magnet is still to be determined but it is expected to be in the range of 5 to 10 degrees. The magnet will be powered by a DC supply during injection. For checkout and tuning of the injection system the magnet will not be powered and the proton beam will be delivered to the downstream beam dump. The beam dump will be capable of handling the total beam power for extended time.

\subsection{Control System}

The control system includes both the hardware and the software necessary to operate the injector. The control system architecture consists of a set of distributed processors, each performing independent functions. Each of the subsystems has a common set of hardware, which influences the writing of a common set of software modules. This approach is cost effective by limiting the amount of software that must be created. The processors that will be selected are off-the-shelf PCs 
and PLCs that offer reliability and are cost effective. The subsystem processors are connected to the injector master control processor via a high-speed data highway network. In addition, provisions for discreet signals routed to each of the processors will be made. The main processors for the system are:

- Injector master control - provides overall control and synchronization with the RCMS master control

- Ion source high voltage processor - located in the high voltage equipment rack for the ion source, it provides local control for the ion source

- Beam transport processor - is responsible for control of the LEBT components and the HEBT components which are magnets, steerers, and diagnostics

- RF system processor - controls RF amplifiers for all of the accelerating cavities

\subsection{Support Subsystems}

The support subsystems for the injector includes cooling water, vacuum, prime power distribution, support structure, and personnel safety.

Cooling Water Subsystem - provides de-ionized cooling water for the ion source, the RFQ, and the DTL, the dipole bending magnet, the RF amplifiers and the vacuum pumps. The subsystem consists of a non-refrigerated recirculating cooler with heat exchange to facility water. Shut-off valves and flow meters are provided for each equipment tap-off. Flow switches are provided for critical components to de-energize them in the event of loss of cooling water.

Vacuum Subsystem - provides the high vacuum pumping for the ion source, LEBT, RFQ, and DTL. The use of cryopumps is incorporated throughout because of their cost effectiveness. A mechanical roughing pump will be provided for initial pump out from atmosphere.

Prime Power Distribution - will receive facility electric power from a single source and distribute to each component requiring it. A fused disconnect will be provided for each powered component. Power to the ion source high voltage equipment rack will be fed through a magnet contactor wired to disconnect power to the rack in the event of loss of cooling water.

Support Structure - this structure provides the physical support and alignment provisions for the entire accelerator and beam transport subsystem. It is made up from aluminum extrusion pieces, which are bolted and or welded together as required. 
Personnel Safety Subsystem - provides personnel safety against hazards in the injector system. Other than facility hazards such as radiation, which must be protected by shielding which is not part of this WBS, the only personnel hazard is the high voltage applied to the ion source rack. To assure personnel protection, a cage at ground potential and an entry door will enclose all items that operate at high voltage. Opening the entry door will result in de-energizing the high voltage rack. 


\section{Synchrotron}

Figure 1 shows the racetrack footprint of the synchrotron, consisting of two straight sections and two 180 degree bend sections or arcs. Each straight section contains five FODO half-cells without dipoles and each arc consists of eight halfcells with dipoles. With a grand total of 26 half-cells, the total circumference of the RCMS is 28.6 meters. Figure 5 shows the optical functions - the beta functions $\beta_{x}$ and $\beta_{y}$ and the horizontal dispersion $\eta$ - for the synchrotron.

\section{Rapid Cycling Medical Synchrotron}

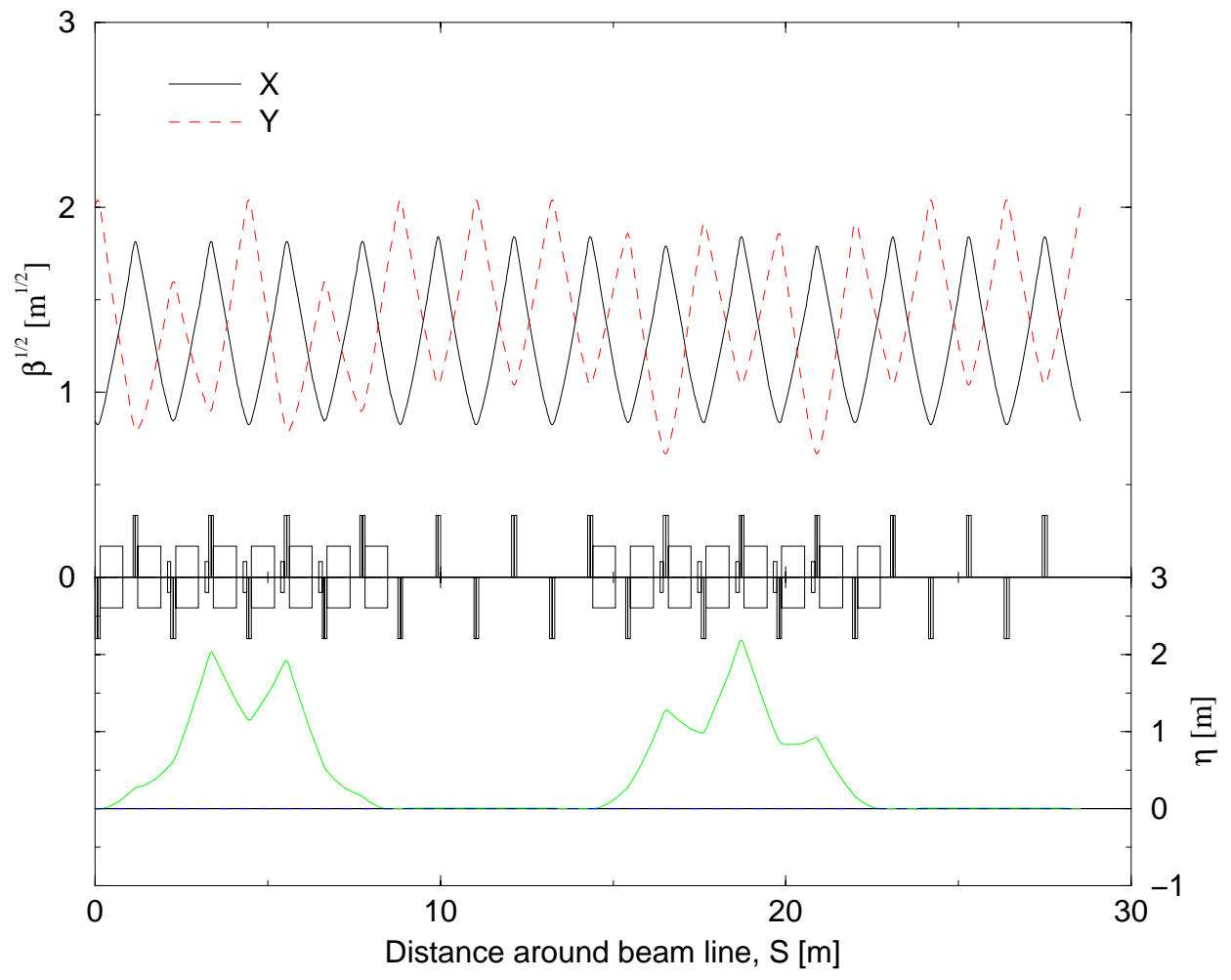

Figure 5: Optical functions for the synchrotron.

The straight sections on each side accommodate the functions of injection, extraction, and acceleration. They are made dispersion free by setting the horizontal betatron phase advance per cell to 90 degrees, so that the net horizontal phase advance per arc is 360 degrees. Two adjacent half-cells on one side are 
occupied by radio frequency cavities. Each side has a fast kicker and a septum magnet separated by one empty half-cell. One straight is used for injection, and the other for extraction. The nominal parameters of the beam injected into the synchrotron are listed in Table 6 .

\begin{tabular}{|lc|}
\hline Property [units] & Value \\
\hline & \\
Injection pulse length, $\Delta t[\mathrm{nsec}]$ & 250 \\
Injection pulse current $[\mathrm{mA}]$ & 2.11 \\
& \\
Normalized rms emittance, $\epsilon[\mu \mathrm{m}]$ & 0.3 \\
Momentum width at injection $(\mathrm{rms}), \sigma_{p} / \mathrm{p}$ & 0.001 \\
Total momentum width, $\Delta p / p$ & \pm 0.0023 \\
Total kinetic energy width, $\Delta K[\mathrm{keV}]$ & \pm 32 \\
& \\
\hline
\end{tabular}

Table 6: Synchrotron beam input parameters.

Variation of the extraction energy is achieved simply by adjusting the extraction time. This potentially avoids the use of energy degraders, delivering higher quality beam with better resolution, but it does not avoid the need to change the excitation of the transport line magnets in proportion to the extraction momentum. However, the transport lines which are described below are in general insensitive to momentum matching errors, since they are achromatic and (mostly) dispersionless.

\subsection{Optics}

Figure 6 illustrates the layout of a bending half-cell, including a quadrupole, a dipole, a Beam Position Monitor (BPM), and a dipole corrector. The RCMS has only one type of each of these elements, minimizing the effort required for component design and development, and reducing the required number of kinds of spares. The dipole is placed as far upstream as possible to maximize the free space available between its downstream end and the next quadrupole. The BPM may be integrated into the vacuum pipe in the center of the RCMS quadrupoles. Table 7 lists the key geometric parameters of the half-cell, and a selection of parameters which summarize the optical functions shown in Figure 5. 


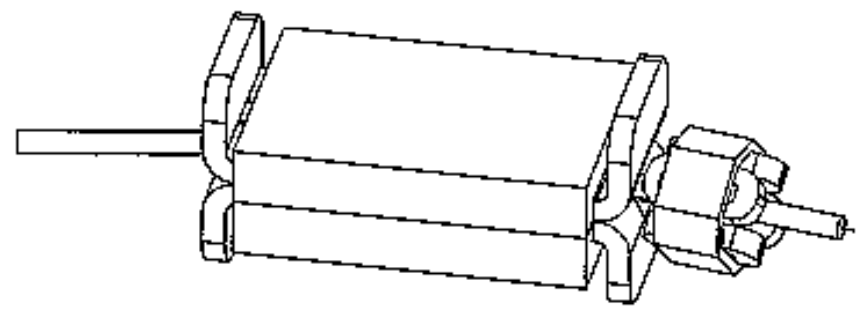

\section{HALF CELL}
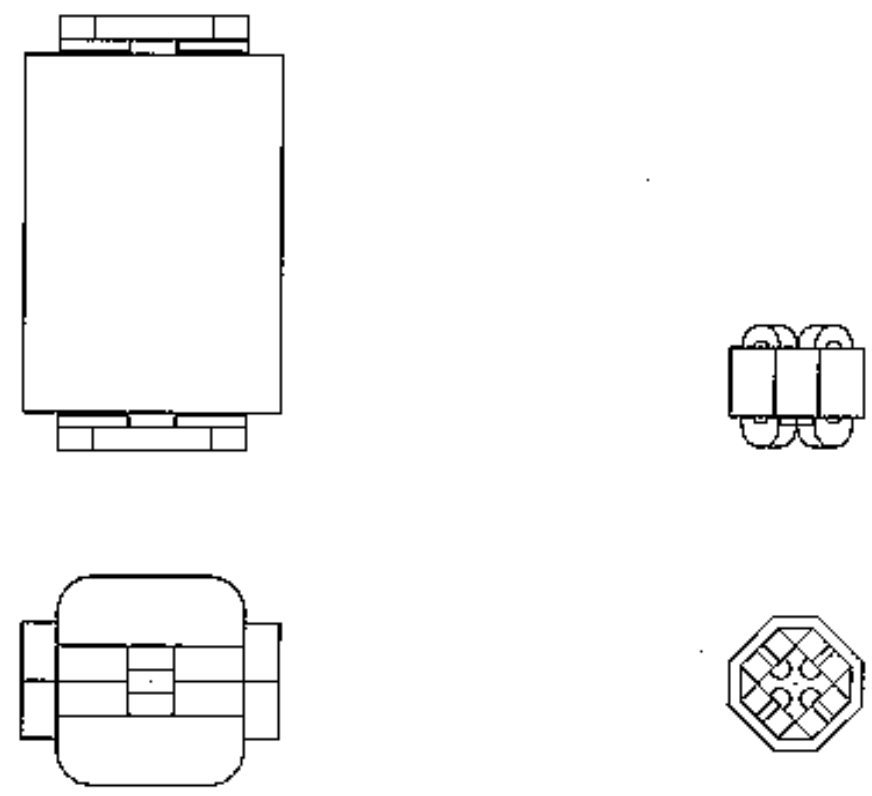

\begin{tabular}{|c|c|c|c|}
\hline $\begin{array}{l}\text { MAGNET } \\
\text { APERTURE } \\
\text { CORE LENGTH } \\
\text { CURRENT } \\
\text { COIL } \\
\text { WEIGHT }\end{array}$ & $\begin{array}{l}\text { RING DIPOLE } \\
4 \mathrm{~cm} \times \mathrm{g} \mathrm{cm}(\mathrm{H} \times \mathrm{h}) \\
63 \mathrm{~cm} \\
2000 \mathrm{~A} \text { (hox) } \\
12 \text { Turns } / \text { Coil } \\
1100 \mathrm{dbs}\end{array}$ & $\begin{array}{l}\text { MAGNET } \\
\text { APERTURE } \\
\text { CORE LENGTH } \\
\text { CURRENT } \\
\text { COIL } \\
\text { WEIGHT }\end{array}$ & $\begin{array}{l}\text { RING QUADRUPOLE } \\
\$ 4 \mathrm{~cm} \\
12 \mathrm{~cm} \\
600 \mathrm{~A} \text { (wox) } \\
6 \text { Turns/Coil } \\
100 \mathrm{lbs}\end{array}$ \\
\hline
\end{tabular}

Figure 6: The FODO half cell and its principal magnets. 


\begin{tabular}{|lc|}
\hline Property [units] & Value \\
\hline & \\
Half-cell length [m] & 1.1 \\
Phase advance per cell, H and V [deg] & 90 \\
Number of FODO cells & 13 \\
& \\
Max. horizontal beta function, $\beta_{x \max }[\mathrm{m}]$ & 3.39 \\
Max. vertical beta function, $\beta_{y \max }[\mathrm{m}]$ & 4.16 \\
Max. dispersion function, $\eta_{\max }[\mathrm{m}]$ & 2.18 \\
Horizontal tune, $Q_{x}$ & 3.25 \\
Vertical tune, $Q_{y}$ & 2.85 \\
Natural horizontal chromaticity, $\xi_{x}$ & -0.88 \\
Natural vertical chromaticity, $\xi_{y}$ & -0.69 \\
Transition gamma, $\gamma_{T}$ & 2.39 \\
\hline
\end{tabular}

Table 7: Optical parameters of the synchrotron.

The horizontal and vertical root mean square beam sizes $\sigma_{x}$ and $\sigma_{y}$ at a given location in the synchrotron are related to these quantities as follows:

$$
\begin{aligned}
& \sigma_{x}=\left(\frac{\beta_{x} \epsilon}{(\beta \gamma)}+\left(\eta \frac{\sigma_{p}}{p}\right)^{2}\right)^{1 / 2} \\
& \sigma_{y}=\left(\frac{\beta_{y} \epsilon}{(\beta \gamma)}\right)^{1 / 2}
\end{aligned}
$$

where the normalized emittance $\epsilon$ is assumed to be the same in both planes, $\sigma_{p}$ is the rms momentum spread, and $(\beta \gamma)$ is a relativistic Lorentz factor. The second term in the brackets is absent in the vertical case because the vertical dispersion is zero. Table 8 lists the maximum expected beam sizes (and other parameters) at the bottom and at the top of the ramp, using the beam parameters from Table 6 and the optical parameters of Table 7 . The contribution of the transverse emittance to the beam size dominates that of the momentum spread.

Since the dipoles are rectangular, their pole face rotations focus the beam only in the vertical plane. This means that the horizontal phase advance in the straight cells is the same as in the bending cells, so that the total horizontal tune is $Q_{x} \approx 3.25$. It also means that the vertical tune is $Q_{y} \approx 4.05$ if the focusing (F) and defocusing (D) quadruples were of the same integrated strength. 


\begin{tabular}{|lcc|}
\hline Property [units] & injection & top \\
\hline & & \\
Kinetic energy, $K[\mathrm{MeV}]$ & 7.0 & 270.0 \\
Momentum, $p[\mathrm{MeV} / \mathrm{c}]$ & 114.8 & 761.3 \\
Lorentz $\gamma$ & 1.0075 & 1.2878 \\
Lorentz $\beta$ & 0.121 & 0.630 \\
Revolution frequency, $F_{\text {rev }}[\mathrm{MHz}]$ & 1.273 & 6.605 \\
Revolution period, $T_{\text {rev }}[\mu \mathrm{s}]$ & 0.785 & 0.151 \\
& & \\
Rigidity, $B \rho[\mathrm{Tm}]$ & 0.383 & 2.539 \\
Dipole field, $B$ [T] & 0.226 & 1.500 \\
Quad field gradient, $G[\mathrm{~T} / \mathrm{m}]$ & 3.52 & 23.32 \\
Quad pole tip field, $B_{\text {pole }}[\mathrm{T}]$ & 0.070 & 0.466 \\
& & \\
Vertical rms beam size, $\sigma_{y}[\mathrm{~mm}]$ & 4.13 & 1.60 \\
Horizontal rms betatron beam size [mm] & 3.73 & 1.44 \\
Horizontal rms momentum beam size $[\mathrm{mm}]$ & 2.18 & 2.18 \\
Total horizontal rms size, $\sigma_{x}[\mathrm{~mm}]$ & 4.32 & 2.61 \\
& & \\
\hline
\end{tabular}

Table 8: Kinematic and beam parameters at the bottom (injection) and top of the acceleration ramp. 
The vertical tune is adjusted by varying the $\mathrm{F}$ and $\mathrm{D}$ quadrupole strengths independently. This can be achieved in practice, with identical quadrupoles, by allowing for two different current taps.

\subsection{Magnets}

There is one rectangular dipole with a magnetic length of 0.665 meters between the quadrupoles in each bending half-cell. Each of the 16 dipoles bends the beam through 22.5 degrees, with a maximum field of $1.5 \mathrm{~T}$. Rectangular dipoles - with parallel end faces and straight sides - are used instead of sector dipoles because they are more easily constructed. More information about the magnets and other aspects of the synchrotron lattice can be found in Table 9 and Figure 6 .

\begin{tabular}{|c|c|}
\hline Property [units] & Value \\
\hline Number of dipoles & 16 \\
\hline Magnetic length of dipole $[\mathrm{m}]$ & 0.665 \\
\hline Max. dipole field (top) [T] & 1.50 \\
\hline Max. dipole $d B / d t[\mathrm{~T} / \mathrm{s}]$ & 60.0 \\
\hline Dipole bend angle [deg] & 22.5 \\
\hline Dipole bend radius [m] & 1.693 \\
\hline Dipole sagitta $[\mathrm{mm}]$ & 32.5 \\
\hline Dipole gap width [mm] & 80 \\
\hline Dipole gap height [mm] & 40 \\
\hline Dipole weight (each) [lbs] & 1100 \\
\hline Number of quadrupoles & 26 \\
\hline Magnetic length of quadrupole [m] & 0.14 \\
\hline F quadrupole gradient (top) $[\mathrm{T} / \mathrm{m}]$ & 23.8 \\
\hline D quadrupole gradient (top) [T/m] & 19.7 \\
\hline Quadrupole pole tip diameter [mm] & 40.0 \\
\hline Quadrupole weight (each) [lbs] & 100 \\
\hline
\end{tabular}

Table 9: Dipole and quadrupole magnet parameters.

The rapid cycling nature of the synchrotron leads to particular, although not unprecedented, challenges in three areas. Two of these areas - the main power supply system and the radio frequency system - are addressed at some length, directly below. 
The third area is in the design of the vacuum chamber pipe through which the beam passes. A conventional solid metal beam pipe cannot be used inside the dipoles, because the eddy currents induced during the ramp would be far too strong. A ceramic beam pipe is a reasonable solution for the quadrupoles and perhaps also for the dipoles. A second option that is used in practice at Cornell and at Fermilab is to put the vacuum chamber around the outside of the dipole. A third option is to use a hybrid vacuum chamber that allows beam image currents to flow along the direction of the beam, but does not allow eddy current loops across the beam pipe. Any one of these three schemes could be made to work. The optimal choice has not been made at the time of writing this pre-conceptual design report.

\subsection{Main power supplies and ramping}

In the initial configuration of the RCMS, a single resonant power supply drives all the dipoles in series. A direct current bias $I_{D C}$ is added to the sinusoidal alternating current $I_{A C}$ to ensure that the minimum current matches the required injection field. The total dipole current $I$ is therefore

$$
I(t)=I_{D C}-I_{A C} \cos \left(2 \pi f_{r e p} t\right)
$$

Injection occurs at $t=0$ when $I=I_{D C}-I_{A C}=300 \mathrm{~A}$. Beam is extracted from the ring sometime before $I=I_{\text {max }}$ at $f_{\text {rep }} t=0.5$, when $I=I_{D C}+I_{A C}$ $=2000 \mathrm{~A}$. This waveform is illustrated in Figure 7. Except for iron saturation effects the beam momentum is directly proportional to the dipole current.

The dipoles comprise the inductive part of a series LC circuit, driven at a resonant frequency that is the same as the repetition rate $f_{\text {rep }}$ of the synchrotron. This is illustrated in Figure 8. Similar resonant power supplies have been used successfully for more than two decades in rapid cycling combined function synchrotrons, such as the Cornell electron synchrotron $(60 \mathrm{~Hz})$ and the Fermilab proton booster $(15 \mathrm{~Hz})$. Such systems are both reliable and economical.

The resonant circuit is driven by two programmable excitation power supplies. In a series resonant topology the excitation power supplies deliver the full magnet current, but at a significantly reduced voltage, when compared to a non-resonant system. Chokes must be placed across each capacitor bank to provide a path for the DC bias current to flow. The chokes are designed with secondary windings which will all be connected in parallel to provide coupling between the individual resonant circuits.

Besides their simplicity, resonant power supplies have the major advantage of "recycling" most of the stored energy between inductors and capacitors. The 


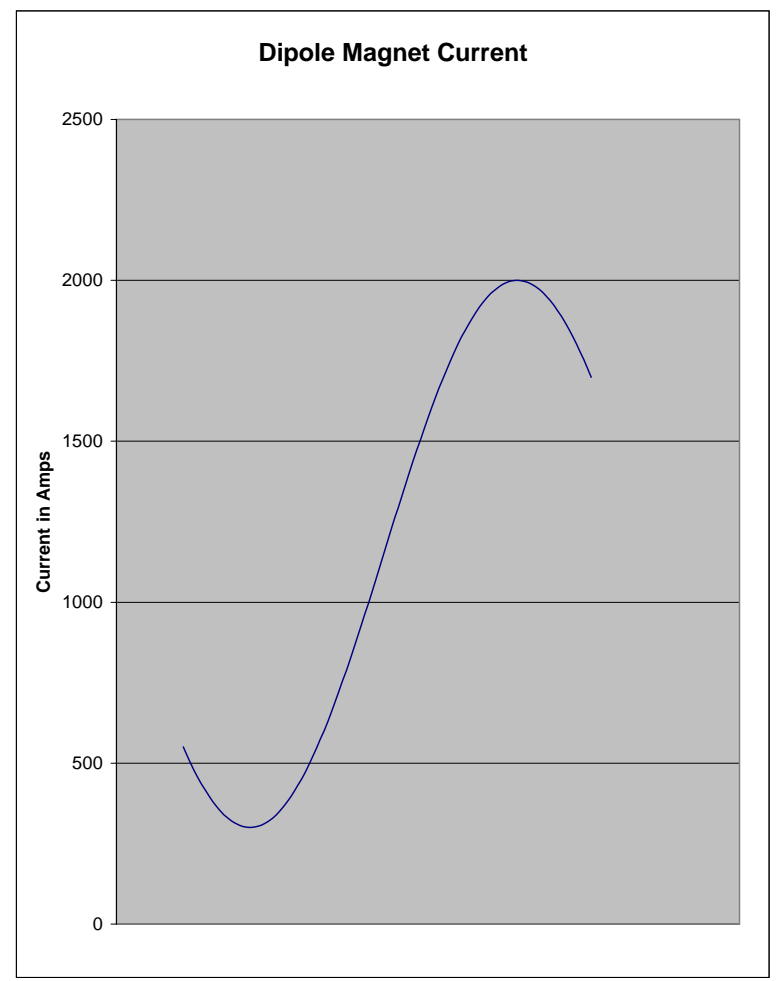

Figure 7: The sinusoidal waveform in RCMS dipoles.

maximum energy stored in the dipoles, when the current and the field are at their maximum values, is approximately

$$
U_{\text {max }}=\frac{B_{\text {max }}^{2}}{2 \mu_{0}}(L W H) N_{d i p}
$$

where $B_{\text {max }}$ is the dipole field, $\mu_{0}=4 \pi 10^{-7}[\mathrm{H} / \mathrm{m}]$ is the permeability of free space, $L, W, H$ are the length, width, and height of the dipole field aperture, and $N_{d i p}$ is the total number of dipoles.

In the approximation that $I_{i n j}<<I_{\text {max }}$, the peak reactive dipole power 


\section{Dipole Series Resonant Power Supplies}

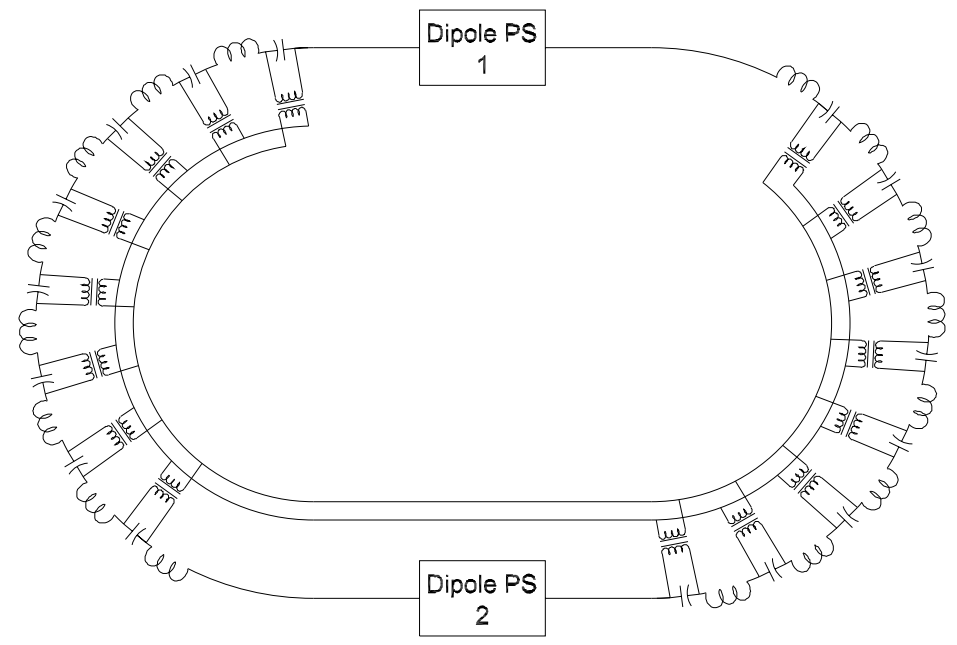

Figure 8: The resonant programmable main dipole power supply system.

$P_{\text {max }}$ is related to $U_{\text {max }}$ through

$$
P_{\text {max }} \approx U_{\text {max }} \frac{3 \sqrt{3} \pi}{4} f_{\text {rep }}=4.08 U_{\text {max }} f_{r e p}=1.86[\mathrm{MW}]
$$

Fortunately the resistive power losses, which need to be continuously replaced by the excitation supplies and the power line, are much less than the reactive power. This makes a resonant system very economical from both a capital equipment and an operational point of view. Table 10 shows that the total of the resistive losses to the dipoles, the choke, and the capacitors is about 182 $\mathrm{kW}$.

The main power supply driving the quadrupoles is much less demanding in power and performance. In order to compensate for field saturation effects in the dipoles (and, to a lesser extent, in the quadrupoles), the quadrupole power supply must be independently programmable. Such a non-resonant power supply is illustrated in Fig. 9. Although non-resonant power supplies are less efficient, the wall plug power necessary to drive the quadrupoles is only $15 \mathrm{~kW}$, almost negligible in comparison to the $182 \mathrm{~kW}$ consumption of the dipole power supply. 


\begin{tabular}{|lc|}
\hline & Value \\
\hline Property [units] & \\
Topology & Series Resonant \\
Repetition rate [Hz] & 15 \\
Number of excitation power supplies & 2 \\
Excitation power supply current [A] & 2000 \\
Excitation power supply voltage [V] & 100 \\
Injection current [A] & 300 \\
Peak current & 2000 \\
Required magnet current, $I_{D C}[\mathrm{~A}]$ & 1150 \\
Required magnet current, $I_{A C}[\mathrm{~A}]$ & 850 \\
& \\
Number of capacitance banks & 16 \\
Number of bypass chokes & 16 \\
Number of dipoles & 16 \\
Capacitance per bank [F] & 0.175 \\
Inductance of choke [mH] & 1.95 \\
Inductance of dipole [mH] & 0.97 \\
Resistance of choke [m $\Omega]$ & 4.6 \\
DC resistance of dipole [m $\Omega]$ & 2 \\
Quality factor & 26 \\
& \\
Stored energy [kJ] & 30.5 \\
Maximum reactive power [MW] & 1.865 \\
Capacitor losses [kW] & 13 \\
Choke losses [kW] & 105 \\
Magnet losses [kW] & 64 \\
TOTAL losses [kW] & 182 \\
& \\
\hline
\end{tabular}

Table 10: Main dipole power supply parameters. 
The quadrupole power supply is a standard switch mode type of unit. Switch mode supplies have the advantage of operating at a high frequency, typically $40 \mathrm{kHz}$, which allows very good regulation and economical filtering. The power supply has a thyristor controlled pre-regulator, which reduces the amount of reactive power the supply draws from the line. This supply is readily available commercially and has a proven reliability.

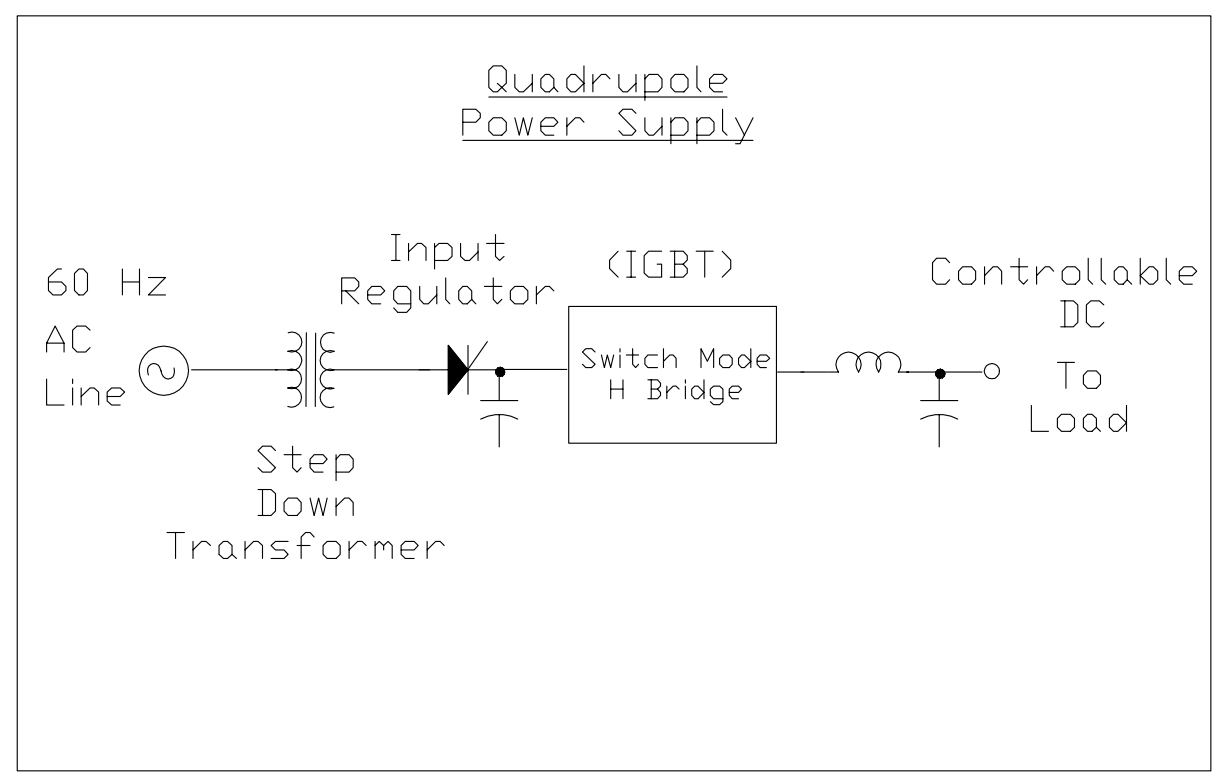

Figure 9: The main quadrupole power supply.

The upgrade path to slow extraction has an important impact on the main dipole power supply, for it, too, must become non-resonant in this scenario. A major advantage of the topology chosen for the resonant dipole power supply is that the excitation power supplies, which are programmable, are rated for the full magnet current. With the reduced ramp rate requirements needed for slow extracted beam, these power supplies can be reconfigured to drive the dipole magnets directly. High power programmable power supplies are in routine operation at the BNL AGS Booster.

The power line swing caused by a resonant power supply is much lower than that due to a comparable rapid cycling programmable power supply; this is a significant advantage in choosing a resonant system. Large variations in 


\begin{tabular}{|lc|}
\hline Property [units] & Value \\
\hline Topology & Switch Mode \\
Repetition rate [Hz] & 15 \\
Number of power supplies & 1 \\
Power supply current [A] & 650 \\
Power supply voltage [V] & 75 \\
Peak Current [A] & 595 \\
& \\
Number of magnets & 26 \\
Inductance of magnet $[\mu \mathrm{H}]$ & 51 \\
DC resistance of magnet [m $\Omega]$ & 1.7 \\
Magnet losses total [kW] & 15 \\
\hline
\end{tabular}

Table 11: Main quadrupole power supply parameters.

reactive power flow cause voltage flicker problems, which can be very costly to solve. This advantage of the resonant system disappears as the ramp rate is decreased.

\subsection{Radio Frequency}

Two ferrite loaded RF cavities provide the voltage needed for acceleration and bunch stability. The unique challenge for these cavities is the very high rate of change of the RF frequency. Typically the resonance frequency of a cavity is changed dynamically to match the changing beam frequency by applying a variable DC bias to the ferrite. However, ferrites have a natural inertia of their permeability which limits the maximum rate of change of frequency. The rate of change that would be required to keep the RCMS cavities on resonance exceeds the value achieved in any previous synchrotron application by a factor of ten.

Fortunately, RCMS cavities do not need to be operated on-resonance, because the voltage required is modest $(\sim 3 \mathrm{kV}$ each) and the beam intensity presents negligible beam loading. To demonstrate the validity of this assertion a laboratory measurement was performed with a spare cavity from the AGS RF system. By reconfiguring the cavity appropriately and using an available RF amplifier it was shown that the necessary RF voltage and frequency change rate can be achieved without dynamically biasing the cavity. Figure 10 shows the test results for the achieved voltage and the required current. A peak voltage of 

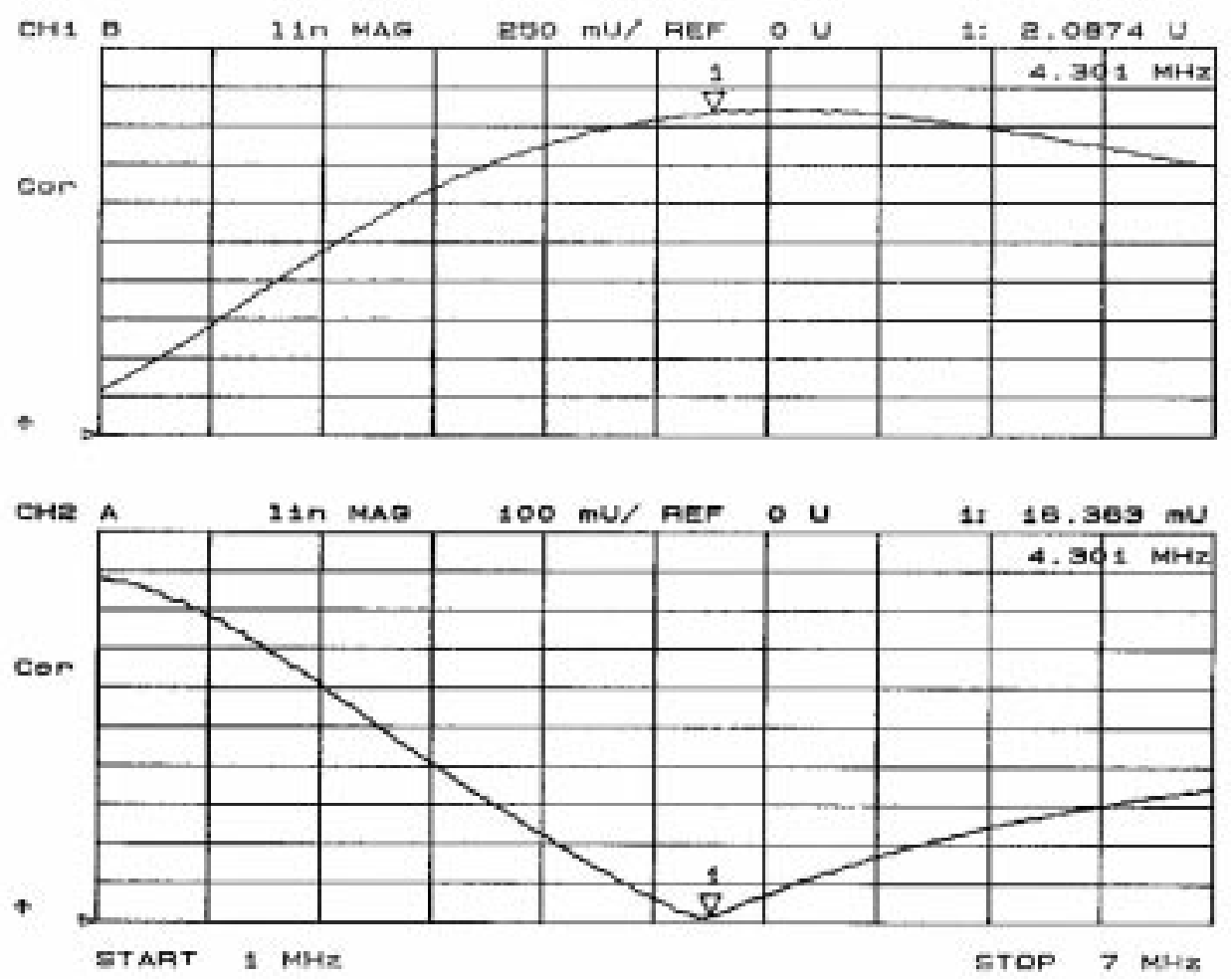

Figure 10: Lab measurement of AGS rf cavity from 1 to $7 \mathrm{MHz}$ with no dynamic tuning. Top is gap voltage, bottom is current from each of two amplifiers, linear scale.

$0.6 \mathrm{kV}$ was attained with acceptable variation of frequency from only 200 Watts of drive power.

These results were used to establish the parameters of circuit model for the cavity/amplifier system, which yielded the results of Fig. 11. From this model a cavity optimized for the RCMS application was developed, by careful choice of the ferrite material and of the geometry of the acceleration gap. The calculated performance of the model RCMS cavity is shown in Figure 12. The calculation shows that the required voltage may be achieved by driving such a cavity with an off-the-shelf solid-state amplifier of less than $5 \mathrm{~kW}$.

The significance of the solid-state amplifier for this project is great. Such an amplifier is completely turn-key in implementation and operation. That is, no RF specialist is required for tune up and maintenance. In contrast, vacuum 
IC) Mas teot Cority
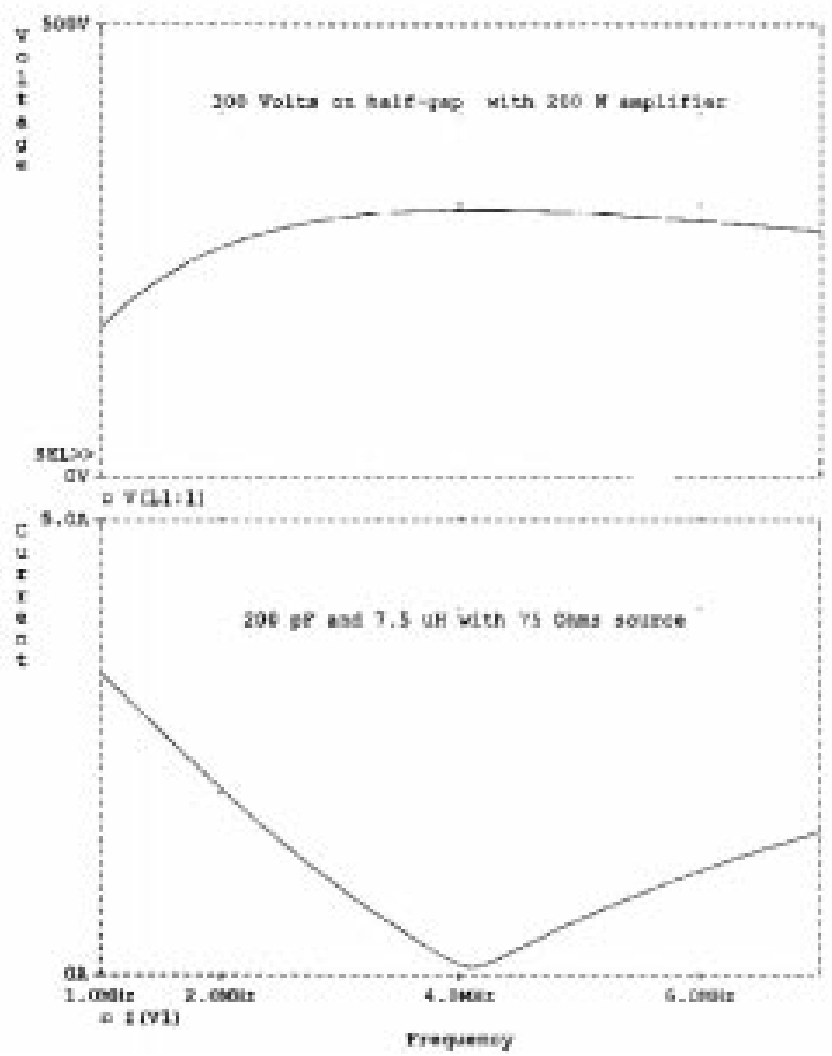

Figure 11: Circuit model calculation of lab measurement, establishing values for parameters of the model.

tube amplifiers need a great deal of ancillary equipment for high voltages and water cooling. They also need periodic maintenance to replace vacuum tubes which have inherently finite life times due to filament failure.

\subsection{Longitudinal dynamics}

In its initial configuration the RCMS ramps the dipole magnetic field from 0.23 $\mathrm{T}$ to $1.50 \mathrm{~T}$ in $33 \mathrm{~ms}$. This leads to a relatively large value for the maximum rate of change of field, $d B / d t=60.0 \mathrm{~T} / \mathrm{s}$. The RF voltage $V_{R F}$ roughly tracks the $d B / d t$ ramp in the nominal RF cycle, keeping the synchronous phase approximately constant throughout acceleration. The relatively low RF voltage which 


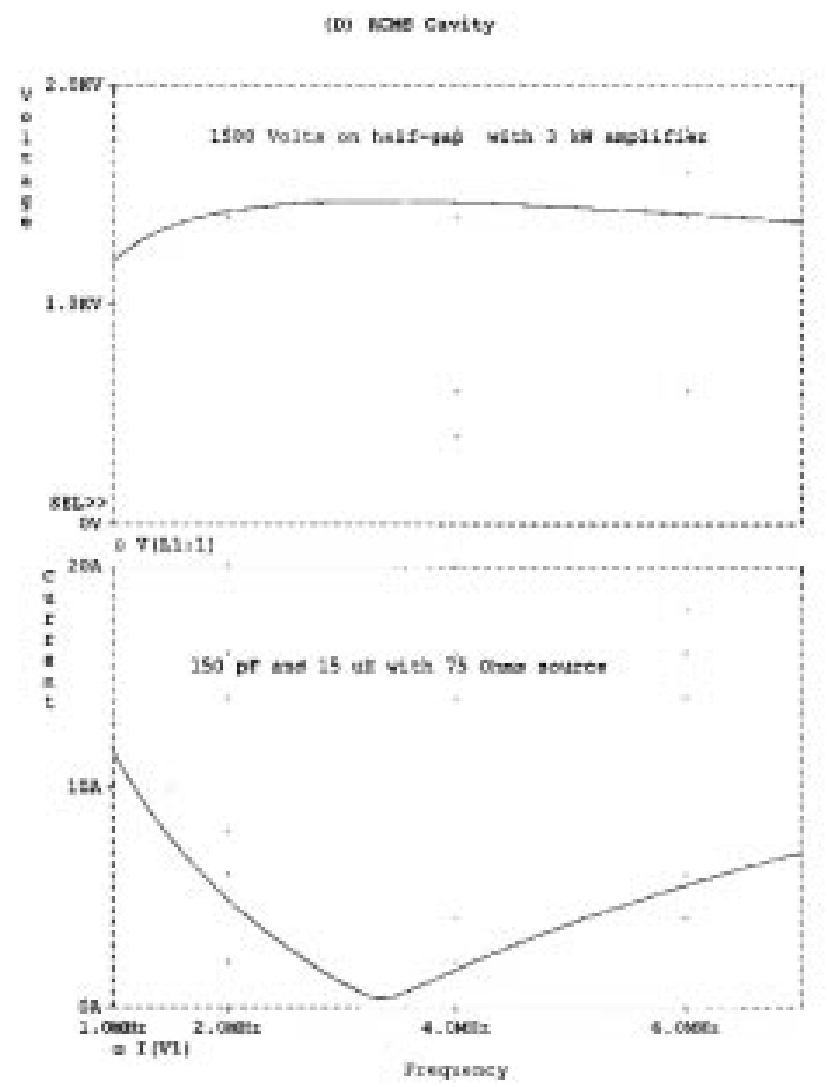

Figure 12: Using the circuit model and optimizing the parameters of the cavity (ferrite material and gap capacitance) the required voltage is achieved with two $2 \mathrm{~kW}$ solid state amplifiers.

is applied at injection implies a low bucket height. The bucket profile at injection matches the given injection beam pulse length and the beam momentum spread, which is naturally small, thanks to the use of an RFQ injector. (The small momentum spread and the small dispersion of the synchrotron lattice also help to reduce the transverse size requirements of the beam pipe and magnets.)

The linac beam profile is an important factor in determining the response of the beam to the RF program. The Drift Tube Linac section of the injector does not include a debuncher section, so that the full kinetic energy spread of the beam is $\Delta K \approx \pm 32 k V$, corresponding to a full momentum spread of $\Delta p / p \approx$ \pm 0.0023 . Simple calculations show that the moving RF bucket is amply large enough to contain this beam at the single turn injection. Simulations of multi- 
particle motion over many synchrotron periods must be applied to accurately determine the evolution of the bunch area and the longitudinal emittance, et cetera.

The simulation results presented in graphical form below correspond to the model parameters shown in Table 12. This table also includes summary results from the simulation.

Figure 13a shows the RF voltage $V_{R F}, d B / d t$, and $B$ for the entire cycle. The RF voltage ramps from $300 \mathrm{~V}$ at injection to a maximum value of $4.5 \mathrm{KV}$ after $15 \mathrm{~ms}$. Figure 14 shows the simulated particle distribution in phase space for the first turn, and then for every 500 turns, up to 5,500 turns, or about 4 ms. The bunch length very quickly increases from its injection value of $250 \mathrm{~ns}$ to to about $350 \mathrm{~ns}$ at $2 \mathrm{~ms}$. This situation is optimized by carefully tuning the injection parameters.
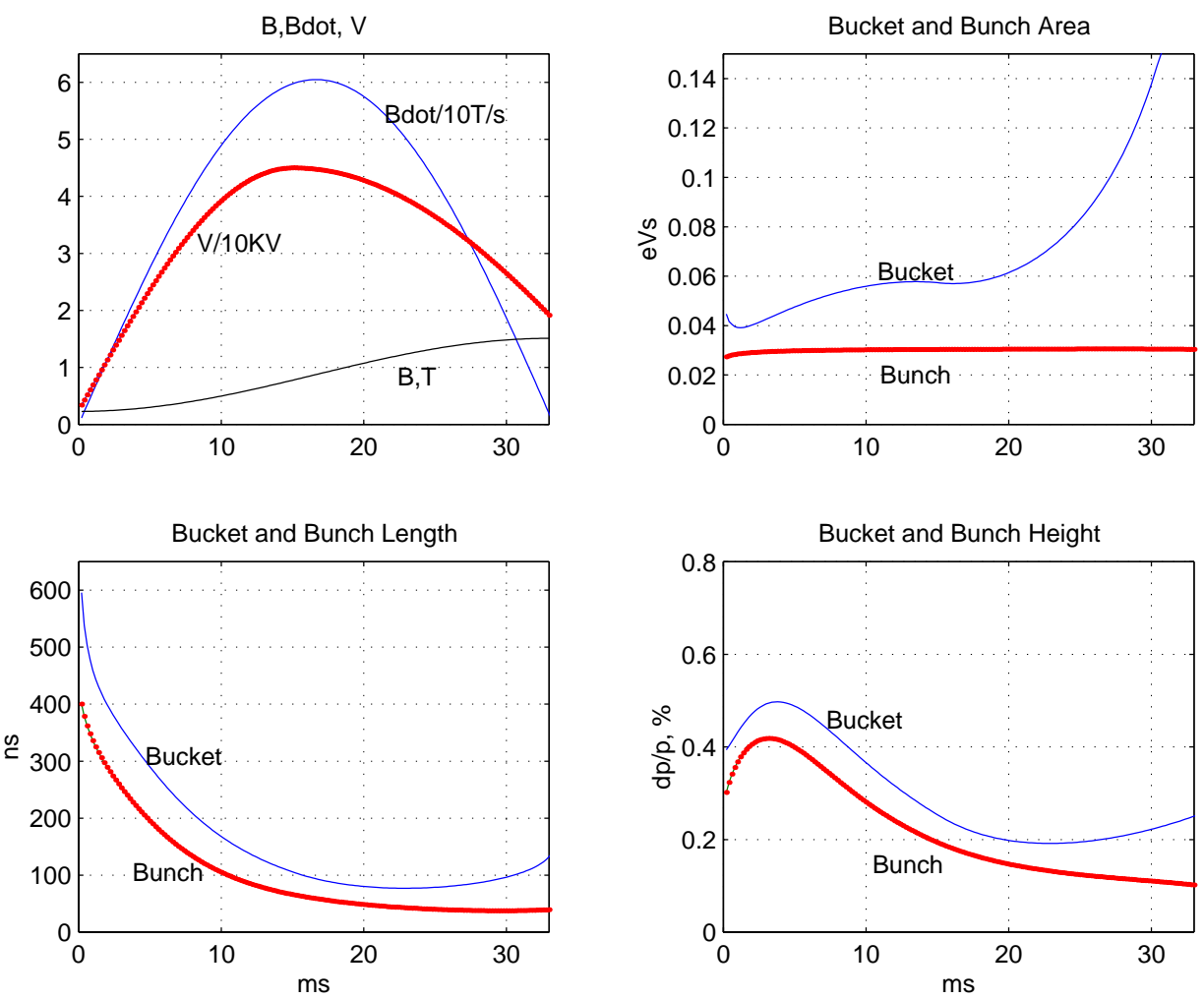

Figure 13: The evolution of longitudinal dynamics parameters during the RCMS acceleration ramp. 


\begin{tabular}{|lc|}
\hline Property [units] & Value \\
\hline Harmonic number, $h$ & \\
Number of cavities & 1 \\
Maximum total voltage, $V_{r f}[\mathrm{kV}]$ & 4.5 \\
Maximum accelerating voltage [kv] & 4.2 \\
Longitudinal emittance (total) [eVs] & 0.03 \\
Normalized rms emittance $[\mu \mathrm{m}]$ & 0.3 \\
& \\
Revolution period (inj), $T_{\text {rev }}[\mu \mathrm{s}]$ & .785 \\
Revolution period (top), $T_{r e v}[\mu \mathrm{s}]$ & .151 \\
Revolution frequency (inj), $F_{\text {rev }}[\mathrm{MHz}]$ & 1.274 \\
Revolution frequency (top), $F_{r e v}[\mathrm{MHz}]$ & 6.605 \\
& \\
Maximum beam momentum spread & \pm 0.0042 \\
Maximum synchronous phase $[\mathrm{degrees}]$ & 41 \\
Maximum synchrotron frequency [kHz] & 6.1 \\
Maximum space charge tune shift, $\Delta Q$ & 0.044 \\
Maximum peak current $[\mathrm{mA}$ ] & 23 \\
Maximum $d f_{R F} / d t$ [MHz/s] & 270 \\
& \\
Minimum bucket length [degrees] & 149 \\
Bunching factor at injection & 0.24 \\
Bunch length at injection [ns] & 400 \\
& \\
\hline
\end{tabular}

Table 12: RCMS Radio Frequency system parameters. 

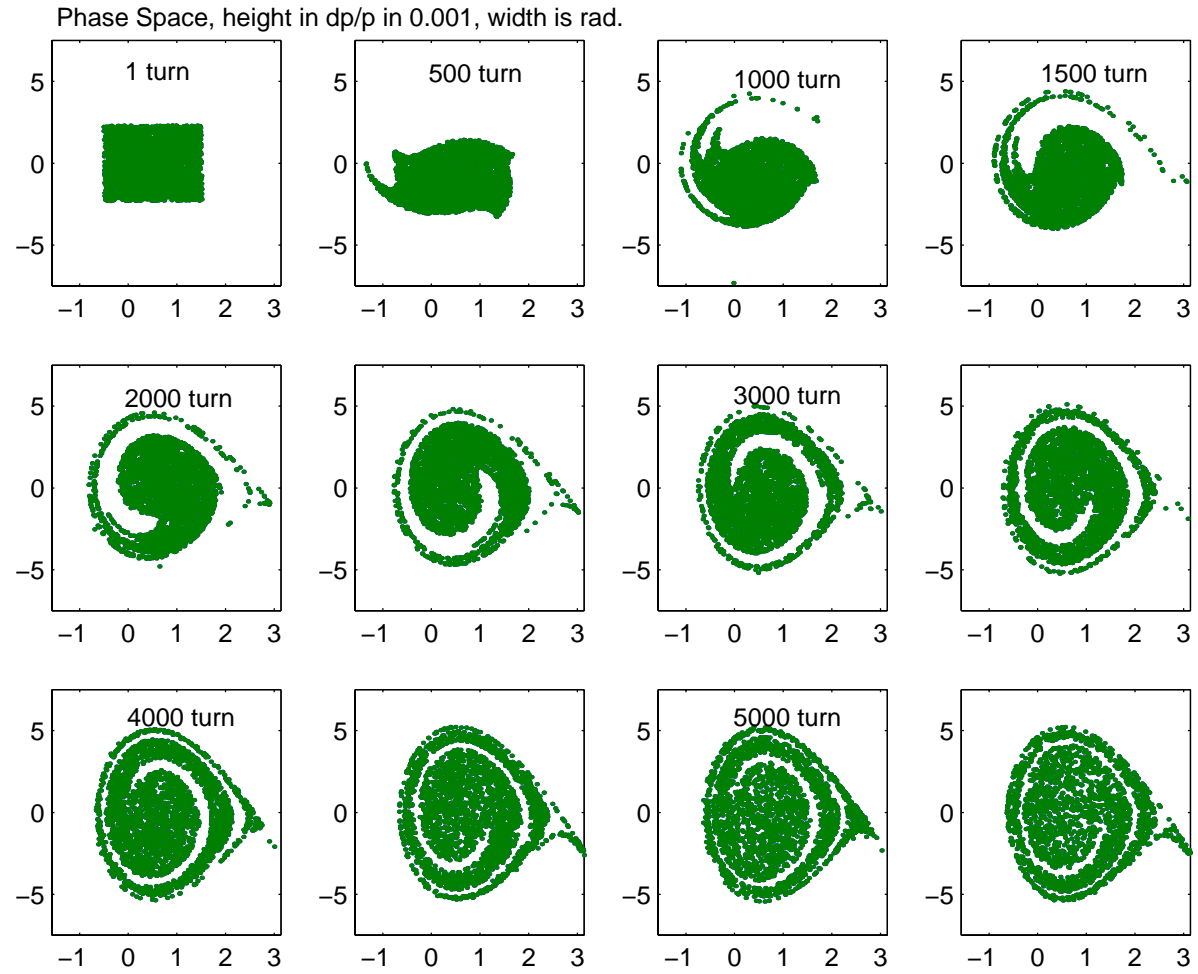

Figure 14: Simulated evolution of the injected beam in longitudinal phase space during the first $4 \mathrm{~ms}$ of acceleration.

The length of the smooth bunch is assumed to be $400 \mathrm{~ns}$ at injection for an analytical calculation whose results show that there is a comfortable margin in the bucket to bunch area ratio, length, and height. Figures $13 \mathrm{~b}, \mathrm{c}$, and $\mathrm{d}$ record these results. Figure 13 shows that there is ample space available in the latter part of the cycle, allowing the RF voltage to be reduced.

Figure 15a shows the space charge tune spread for a normalized rms transverse emittance of $\epsilon=0.3[\mu \mathrm{m}]$, the value at the output of the DTL. Figure 15b shows the maximum beam radius, horizontally and vertically. The largest beam size, at injection, is $4.3 \mathrm{~mm}$. Finally, Figures $15 \mathrm{c}$ and $\mathrm{d}$ show the RF frequency and synchrotron frequency as a function of time. 

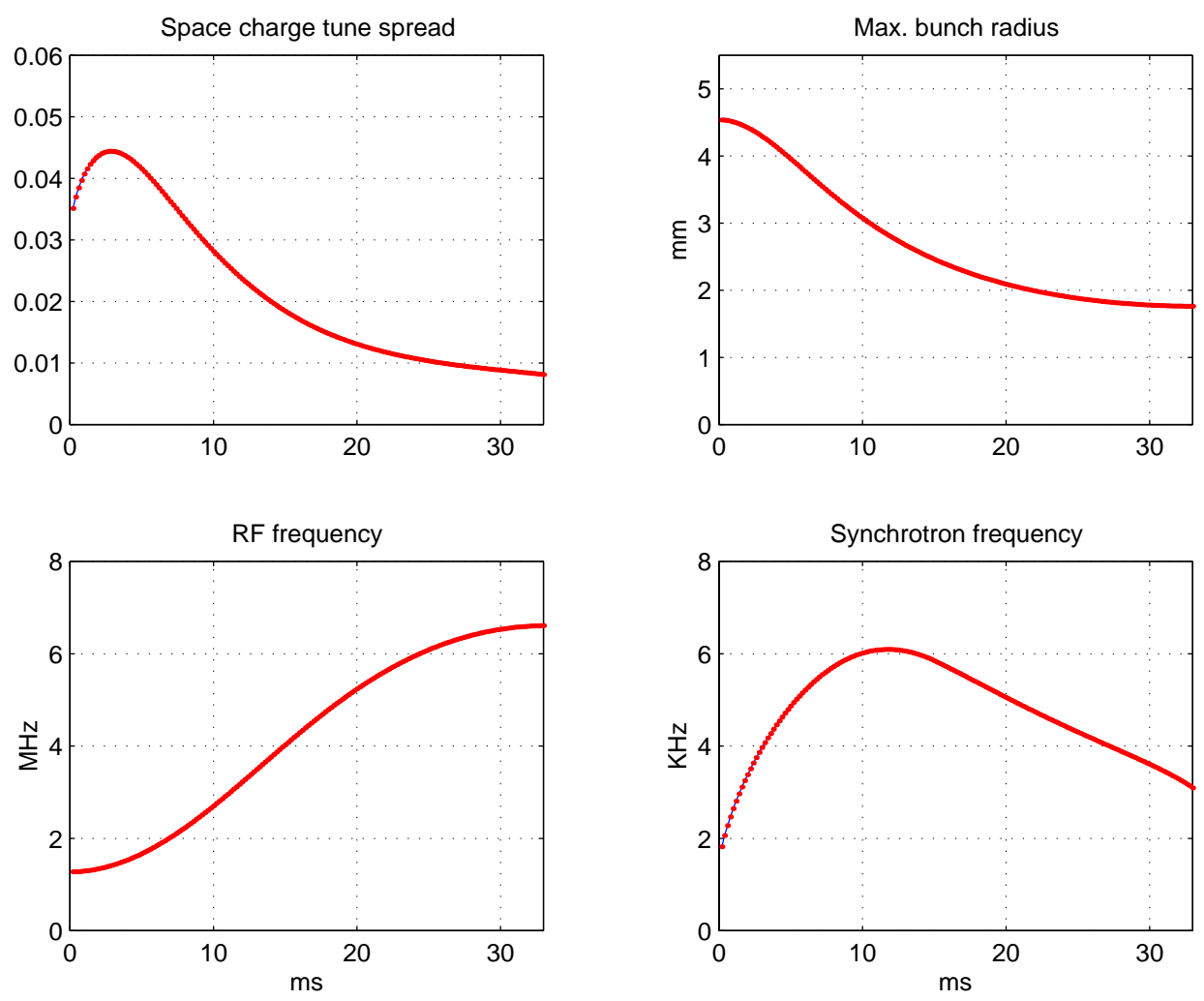

Figure 15: The evolution of more longitudinal dynamics parameters during the RCMS acceleration ramp. 


\subsection{Accelerator controls}

The principal hardware items which appear to be unique in controls required for a synchrotron-based system are probably only two, both related to the fastchanging time dependence of magnet and other ramped equipment. A distributed, microsecond resolution timing link, preferably using encoded signals would be required for scheduling synchrotron cycle actuations. In addition, fast multi-setpoint waveform generators and digitizers would be required for programmed magnet and rf control. BNL has considerable experience with both items and could contribute specifications and/or designs for such items.

The main area in which BNL could be expected to contribute to the controls software for running the facility would be with high level control algorithms for automation of synchrotron setup and operation and expert diagnostics. 


\section{Beam delivery}

This section describes three optical components of the RCMS design, through which beam passes before reaching the patient:

1. A variable geometry "switch yard and transport line" section, which takes the beam from the synchrotron and directs it towards one of the patient treatment rooms. The optical design of the simplest and most minimal delivery system - a fixed horizontal beam line with a conventional double scattering nozzle - differs little from the transport line that feeds it, except in the immediate vicinity of the nozzle.

2. A "planar gantry" system that enables beam delivery to the patient from any direction around the gantry axis. The solution which is presented implicitly assumes that space constraints do not force the adoption of a corkscrew geometry. The distance from the end of the gantry to the patient is adjustable (in the design stage).

3. A "variable focusing nozzle" magnetically shapes the round spot size of the beam at the patient over a wide dynamic range of almost two orders of magnitude, always with a converging beam. This nozzle can be used either at the end of a fixed beam line, or at the end of a gantry.

All transport lines and gantries discussed here are constructed from the same dipoles and quadrupoles used in the synchrotron itself.

\subsection{Switch yard and transport line}

Figure 16 shows the schematic layout of a horizontal beam transport line - or fixed horizontal beam line - which extracts protons from the synchrotron and delivers them over approximately 34 meters (a distance that is easily adjusted) to the patient. The figure shows the behavior of the optical functions in one configuration of the entire line. Table 13 lists various parameters of the transport line.

The first two half-cells of the transport line shown here are the same two half-cells that begin the extraction straight in the synchrotron, starting with a D quadrupole. Immediately after the D quadrupole is a fast kicker that deflects the beam vertically by a small angle of approximately 6.0 milliradians. This causes the beam to be displaced by about 24 millimeters at the location of a horizontally bending Lambertson septum magnet one cell downstream. A full-energy kicked beam experiences a field of about $1.2 \mathrm{~T}$ in the $0.4 \mathrm{~m}$ long Lambertson, giving it a horizontal angle of approximately 0.178 radians, and displacing the beam horizontally by 170 millimeters at the next quadrupole 


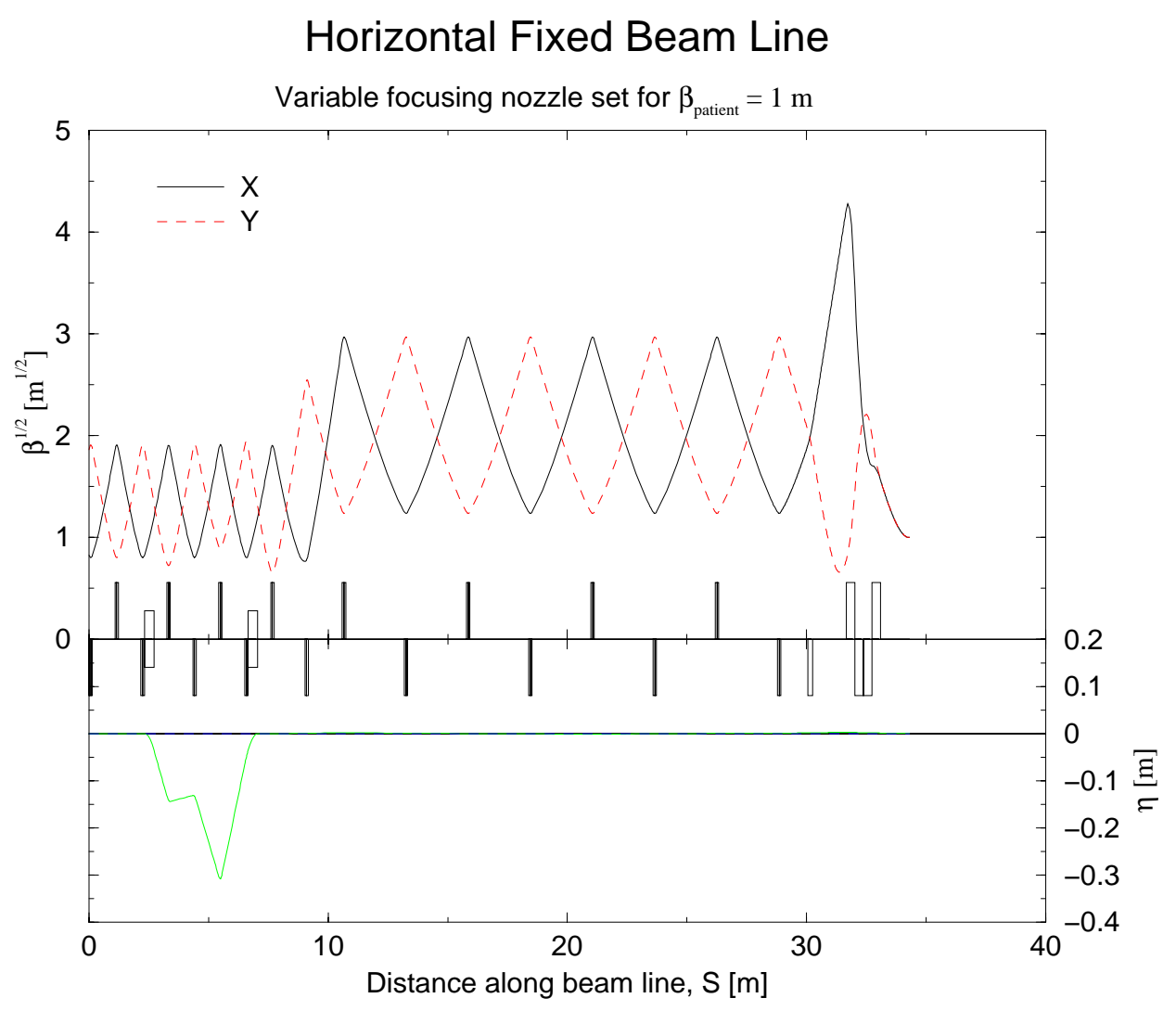

Figure 16: Optical functions through extraction, switch yard, transport line, and variable focusing nozzle, in a sample horizontal fixed beam line.

downstream. The extracted beam is physically separate from the RCMS at this stage but is still traveling in a FODO cell structure like that of the ring.

Both the horizontal and the vertical extraction angles launch dispersion waves in the transport line, but the vertical dispersion is negligible small. The horizontal dispersion wave can be canceled by placing a dipole with the same bend angle as the Lambertson $4 N+2$ full cells downstream, where $N$ is any integer, or by placing an equal and opposite bend $4 N$ full cells downstream. In the case presented here, a bend of the same polarity is placed 2 full cells downstream, about 5.2 meters downstream from the extraction Lambertson. A switch yard is easily constructed to serve as many dispersion free transport lines as desired. The ability to add treatment lines in the future is guaranteed, so long as floor space remains available. 


\begin{tabular}{|lc|}
\hline Property [units] & Value \\
\hline Kicker bend angle [radians] & 0.006 \\
Lambertson bend angle [radians] & 0.178 \\
Magnetic length of Lambertson [m] & 0.4 \\
Maximum Lambertson field [T] & 1.2 \\
Length of transport line half-cells [m] & 2.6 \\
\hline
\end{tabular}

Table 13: General parameters of the horizontal delivery line.

Short half-cells are desirable in the synchrotron since the beta functions are proportionally small, leading to tighter focusing and a smaller beam. This allows the use of magnets with small apertures and small transverse dimensions in general. The tight focusing of the synchrotron can be relaxed in the transport lines (by spacing the same quadrupoles further apart) because the beam size at even the lowest extraction energy is much smaller than the beam size at injection.

If the quadrupoles in the transport line are placed twice as far apart as normal, the beta functions are twice as large, and the beam size is $\sqrt{2}$ times as large (at the same momentum). The quadrupole excitation current is also halved, so the power consumption per magnet is one quarter of nominal. Since there are half as many quadrupoles per meter of transport, the power consumption per meter is only one eighth of the amount that would be required if the nominal half-cell length were used.

The "lengthener" part of the transport line smoothly matches the optical functions of nominal length synchrotron FODO cells to the double length transport FODO cells. The height of a box representing a quadrupole in Figure 16 is proportional to the strength of that quadrupole, showing that the three "lengthener" quadrupoles each have different strengths. These three quadrupoles must have separate power supplies, or shunts.

Again it should be emphasized that the design presented here is pre-conceptual, and has not undergone parameter optimization. For example, the optimum length of the long half-cells is probably not exactly twice the RCMS half-cell length. 


\subsection{Planar gantry}

The planar gantry is a simple structure using standard synchrotron magnets. Its optics are shown in Figure 17, and its geometry in Figure 18. Thus, the gantry is light and economical in construction and operation.

The optics are fully matched, achromatic, and flexible. The distance between the end of the gantry and the isocenter is a variable which is set at design time. Values in the range from $1.2 \mathrm{~m}$ to $3.7 \mathrm{~m}$ are trivially achievable. In the design presented here a nominal value of $3 \mathrm{~m}$ is assumed to be sufficient to insert a conventional double scattering system, and for a scanning upgrade.

\section{Gantry Optics}

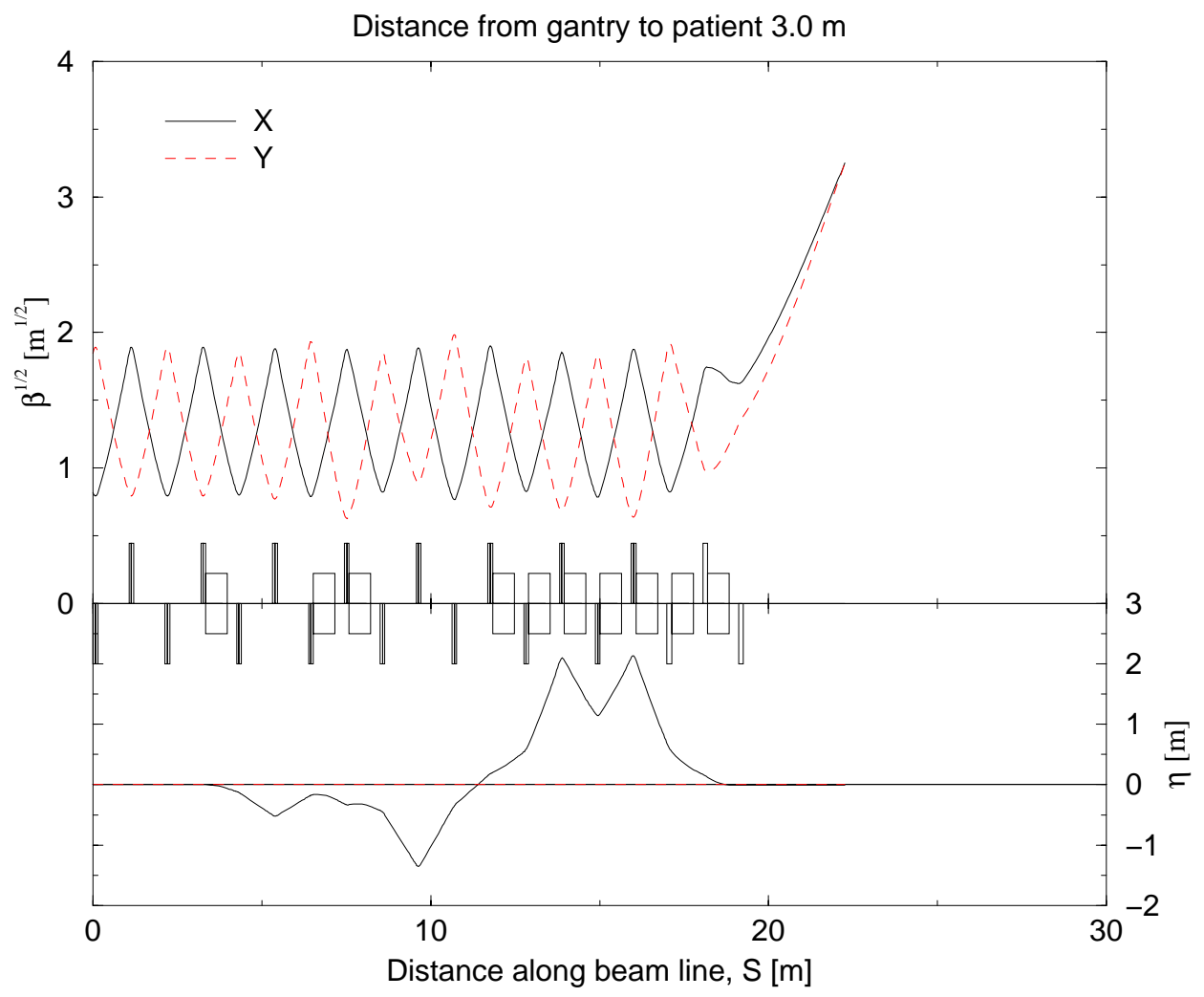

Figure 17: Fully matched achromatic strongly focused optical functions through the planar gantry. 


\section{Gantry Geometry}

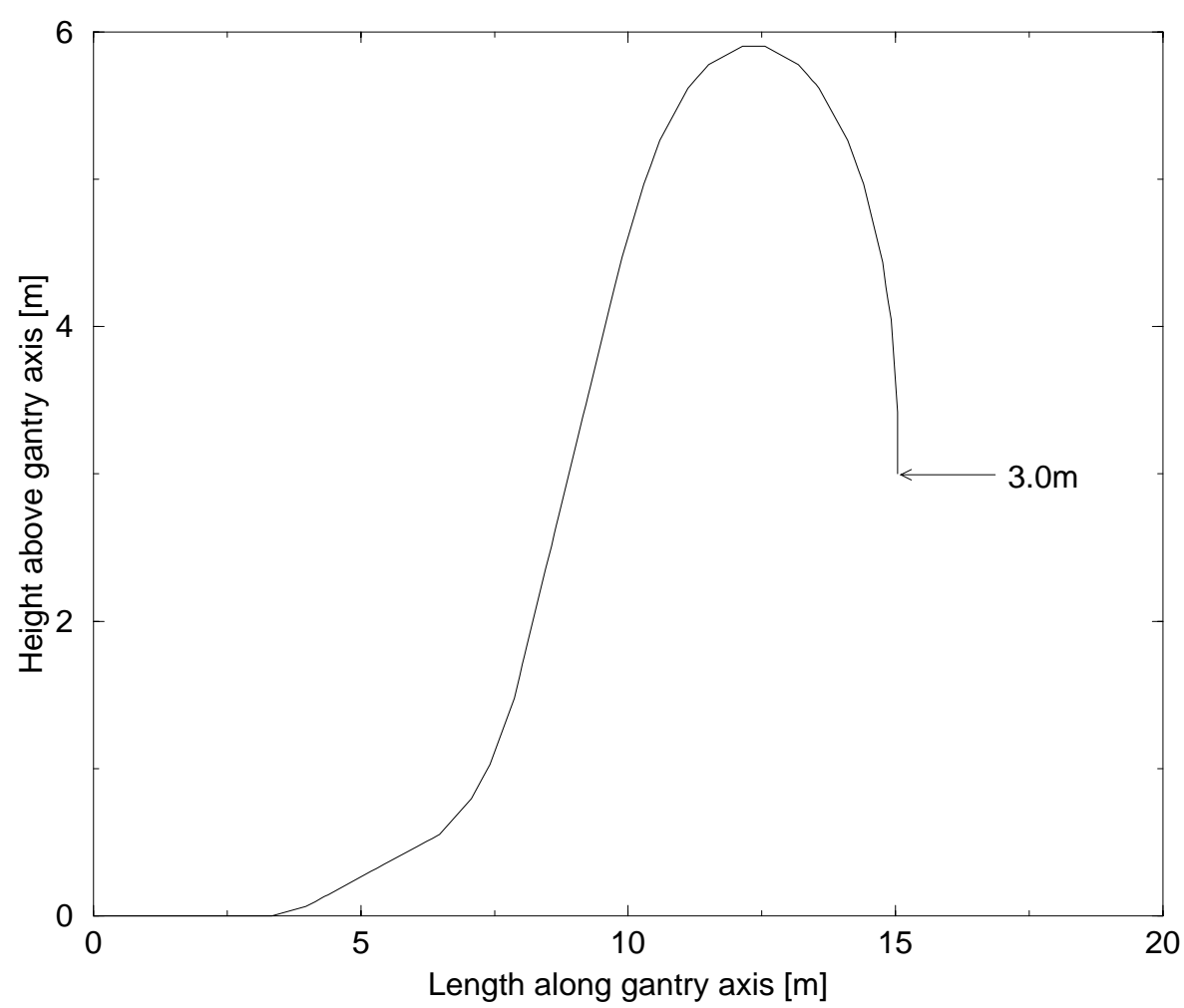

Figure 18: Geometry of the planar gantry, showing a radius of just under 6 meters.

\subsection{Variable focusing nozzle}

Figure 19 shows the optical performance of the variable focusing nozzle as the beta function at the patient, $\beta_{\text {patient }}$, is adjusted over three orders of magnitude, from 0.1 to 100 meters. In the absence of multiple scattering in the patient, this is equivalent to beam spot sizes varying from 0.35 to 11.1 millimeters at 270 $\mathrm{MeV}$. When $\beta_{\text {patient }}$ is very small, the beam is strongly convergent, significantly reducing the entrance radiation dose. However, the smallest practical beam size is limited by multiple scattering, so that such sharp focusing is not advantageous except for shallow problems such as ocular tumors. When $\beta_{\text {patient }}$ is very large, the practical limit to the maximum size of the beam is the aperture of the triplet quadrupoles. In this configuration the beam is barely convergent - it 
is essentially parallel. Note that in no case can the beam be considered to be coming from a point source; hence there is no inverse-square augmentation of entrance dose, and the SAD is always negative.
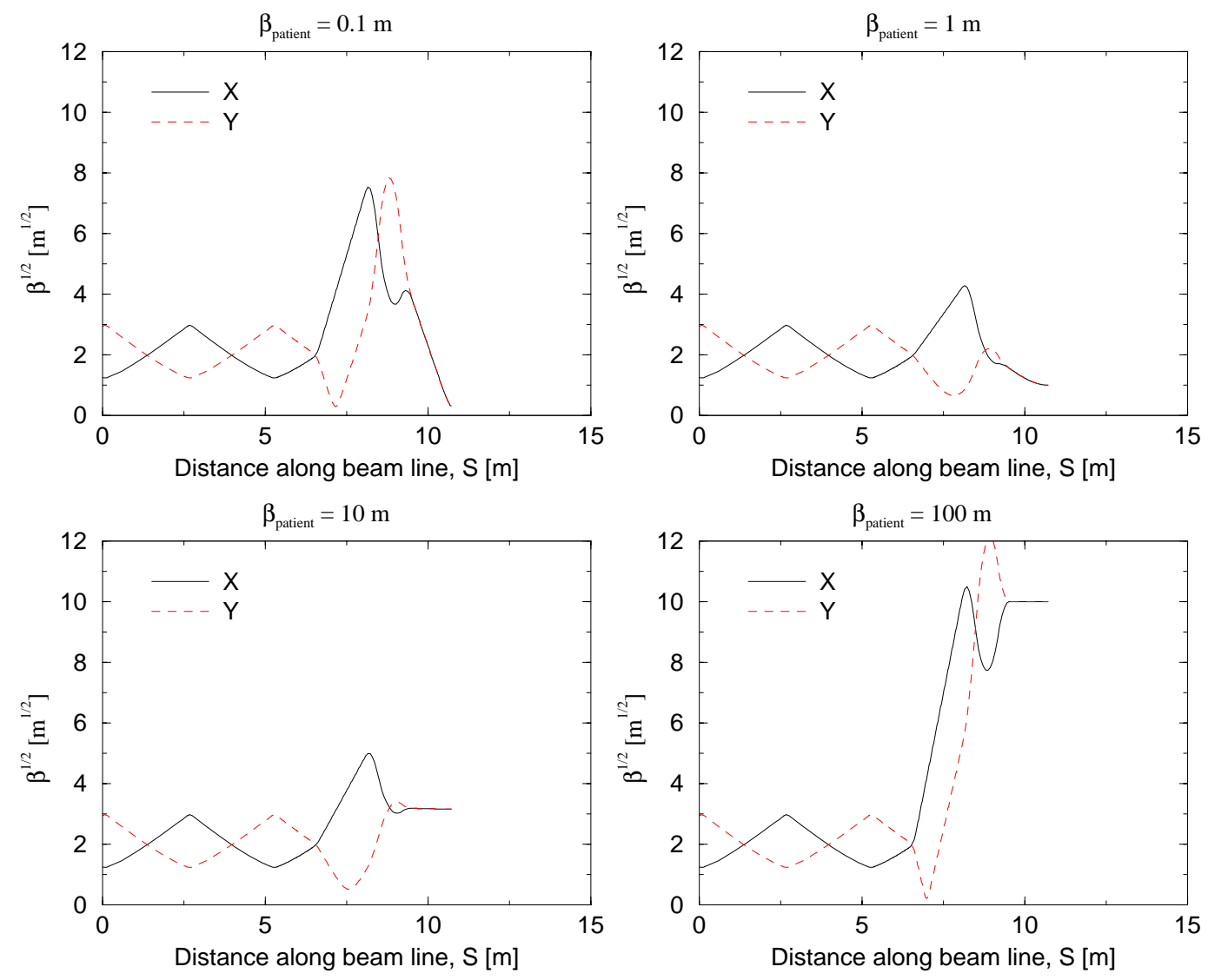

Figure 19: Optical functions of the variable focus nozzle at four settings, showing a dynamic range of 1000 in $\beta_{\text {patient }}$, with values from $0.1 \mathrm{~m}$ to $100 \mathrm{~m}$.

Table 14 shows the excitations of the four nozzle quadrupoles, as measured by the slope of the B-field, in the four configurations shown in Figure 19. The table shows that quadrupoles Q1 and Q4, which undergo the largest variations, must have separate power supplies. It may be possible to power quadrupoles Q2 and Q3 using a single power supply and a shunt. If the maximum acceptable quadrupole pole tip field is $1.5 \mathrm{~T}$ when the field gradient is 13.4 Tesla per meter, then the pole tip diameter of the triplet quadrupoles must be less than 224 millimeters. This is much larger than the largest beam size of 16 millimeters 
that occurs when the $70 \mathrm{MeV}$ beams are essentially parallel, so that the beam has the same size in the triplet as in the patient.

\begin{tabular}{|lcccc|}
\hline & 0.1 & 1.0 & 10.0 & 100.0 \\
\hline$\beta_{\text {patient }}[\mathrm{m}]$ & & & & \\
\hline Focal spot size @ $70 \mathrm{MeV}$ [mm] & 0.50 & 1.59 & 5.04 & 15.9 \\
Focal spot size @ 270 MeV [mm] & 0.35 & 1.11 & 3.51 & 11.1 \\
Gradient of Q1 [T/m] & 10.3 & 3.5 & 1.7 & 5.0 \\
Gradient of Q2 [T/m] & 10.5 & 8.4 & 5.6 & 6.7 \\
Gradient of Q3 [T/m] & 13.4 & 12.2 & 9.8 & 9.9 \\
Gradient of Q4 [T/m] & 17.4 & 4.6 & 7.4 & 28.1 \\
& & & & \\
\hline
\end{tabular}

Table 14: Four focusing configurations of the nozzle. The spot sizes quoted do not include the effects of multiple scattering. Except for the first row of the table, all values are quoted for a kinetic energy of $270 \mathrm{MeV}$, and an rms emittance of $\epsilon=1.0[\mu \mathrm{m}]$.

\subsection{Treatment planning and patient safety}

The goals of patient treatment planning are conceptually simple: to deliver the intended dose to the treatment volume while minimizing damage to normal tissue and avoiding damage to critical anatomical structures. However, achieving these goals in a realistic three-dimensional situation of an irregularly shaped tumor surrounded by anatomical structures of variable density and composition is a challenge to computing and control systems. Fully realizing the potential of proton therapy requires an integrated approach to medical imaging, patient treatment planning in three dimensions, patient localization, and control of accelerator and beam delivery systems using state-of-the-art computing techniques. Digital dose delivery holds the most promise for achieving these goals.

A rapid cycling synchrotron lends itself to a simple, reliable approach to patient safety; this is one of its main virtues. Conditions will be monitored on each cycle; if dangerous abnormal conditions are detected, any beam currently in the synchrotron will be aborted and subsequent cycles will be inhibited at the source. An abort system internal to the synchrotron is also appropriate. Two dipole correctors separated by 180 degrees of phase advance in one of the arcs can be pulsed to sweep the beam into an absorber block halfway between them. The trigger to the extraction kicker would simultaneously be inhibited. 
Examples of situations that would produce this response include an unexpectedly large proton bunch intensity, out of tolerance orbit fluctuations, errors in gantry or patient alignment, or a hardware failure in the final beam steering system. Detection of alarm conditions will require continuous monitoring of beam position and circulating-beam intensity monitors. The final intensity monitor will also serve to $\log$ beam delivery automatically for the patients records. The record will contain pulse-by-pulse information of exposure and orientation so that dosages can be reconstructed in complete detail.

One can imagine rare but perhaps not impossible failure modes that conspire to circumvent such monitoring systems. A great safety advantage of using a rapid cycling synchrotron is that the maximum single bunch intensity is too low to cause serious damage even if a single bunch goes astray and strikes the patient in an unexpected way. As a last line of defense in a multi-layered approach to patient safety, monitors just upstream of the patient will detect the first such out-of-tolerance bunch, and the beam will be inhibited at the source on subsequent cycles until the situation is understood. Our goal is to provide safe, precise, effective, thoroughly monitored treatment using highly reliable, easily maintained (or replaced) hardware. 